\title{
Well-posedness for systems of time-dependent hemivariational inequalities in Banach spaces
}

\author{
Lu-Chuan Ceng ${ }^{a}$, Yeong-Cheng Liou ${ }^{b, c, *}$, Jen-Chih Yao ${ }^{d}$, Yonghong $\mathrm{Yao}^{\mathrm{e}}$ \\ ${ }^{a}$ Department of Mathematics, Shanghai Normal University, Shanghai 200234, China. \\ ${ }^{b}$ Department of Healthcare Administration and Medical Informatics, Center for Big Data Analytics \& Intelligent Healthcare, and \\ Research Center of Nonlinear Analysis and Optimization, Kaohsiung Medical University, Kaohsiung 807, Taiwan. \\ ${ }^{c}$ Department of Medical Research, Kaohsiung Medical University Hospital, Kaohsiung 807, Taiwan. \\ ${ }^{d}$ Center for General Education, China Medical University, Taichung 40402, Taiwan. \\ ${ }^{e}$ Department of Mathematics, Tianjin Polytechnic University, Tianjin 300387, China.
}

Communicated by Y.-B. Xiao

\begin{abstract}
In this paper, we generalize the concept of $\alpha$-well-posedness to a system of time-dependent hemivariational inequalities without Volterra integral terms in Banach spaces. We establish some metric characterizations of $\alpha$-well-posedness and prove some equivalence results of strong $\alpha$-well-posedness (resp., in the generalized sense) between a system of time-dependent hemivariational inequalities and its derived system of inclusion problems. (C)2017 All rights reserved.
\end{abstract}

Keywords: System of time-dependent hemivariational inequalities, $\alpha$-well-posedness, monotonicity, Clarke's generalized gradient, regularity.

2010 MSC: 49K40, 47J20, 49J52.

\section{Introduction}

In 1983, Panagiotopulos [27] introduced and studied the hemivariational inequality concerning nonsmooth and nonconvex energy functions, which is an important generalization of variational inequality. As a useful tool, this type of inequalities and their systems have been used to describe many important problems arising in mechanics and engineering, such as unilateral contact problems in nonlinear elasticity, thermoviscoelastic frictional contact problems, and obstacles problems, see, e.g., [25, 26, 28]. By using the generalized directional derivative (in the sense of Clarke) and the Clarke's generalized gradient (see definitions in Section 2), many kinds of hemivariational inequalities and systems of hemivariational inequalities, including stationary hemivariational inequalities, evolutionary hemivariational inequalities and their systems, etc., have been studied by many researchers in recent years. We can refer to, e.g., $[1,2,6,12,24-26,28,34,37-39]$ and the references therein for more details.

\footnotetext{
*Corresponding author

Email addresses: zenglc@hotmail.com (Lu-Chuan Ceng), simplex_liou@hotmail.com (Yeong-Cheng Liou), yaojc@mail.cmu.edu.tw (Jen-Chih Yao), yaoyonghong@aliyun.com (Yonghong Yao)

doi:10.22436/jnsa.010.08.26
} 
There are two important approaches to study the existence and uniqueness of solution to various types of hemivariational inequalities in the recent literature on hemivariational inequalities. One is closely related to Knaster-Kuratowski-Mazurkiewicz theorems and fixed point theorems, which are used by Panagiotopulos et al. [29], Repovs and Varga [30], Costea and Radulescu [10], and Zhang and He [41] to study stationary hemivariational inequalities and systems of stationary hemivariational inequalities. The other is closely related to surjectivity theorems concerning pseudomonotone and coercive operators, which are captured by Migorski et al. [24], Carl et al. [1], Naniewicz and Panagiotopulos[26], Xiao and Huang [33], and Liu [22] for various types of stationary hemivariational inequalities and evolutionary hemivariational inequalities. Before that, Panagiotopulos formulated two systems of hemivariational inequalities for the behavior of von Karman plates and linear thermoelastic materials in Chapter 7.3 of monograph [28], respectively. However, the regularity hypotheses on the multi-valued terms were quite unnatural for the existence of solutions to the two systems of hemivariational inequalities. Very recently, Xiao et al. [35] studied a system of time-dependent hemivariational inequalities with Volterra integral terms by using a surjectivity theorem for pseudomonotone and coercive operators, and the Banach fixed point theorem for contraction mappings. Under some appropriate conditions, the existence and uniqueness result of solution to the problem considered was established by proving that a derived vector inclusion problem with Volterra integral term is solvable.

On the other hand, Tykhonov [31] first introduced and considered the well-posedness for a minimization problem in 1966, which not only is one of the classical concepts in the optimization theory but also has a profound impact on the development of optimization problems and their related problems. After that, various kinds of results concerned with well-posedness for many optimization problems were established and various kinds of well-posedness for optimization problems and their related ones were studied extensively in recent years by a large number of researchers in many fields; see e.g., [8, 15, 21, 42] and the references therein. In 1981, Lucchetti and Patrone [23] extended the notion of well-posedness for optimization problems to a variational inequality for the first time. By using Ekeland's theorem, they gave a characterization of Tykhonov's well-posedness for a minimizing problem with a convex lower semi-continuous function on a closed convex set. Since then, various kinds of well-posedness for optimization problems, such as LP well-posedness and generalized well-posedness etc., are extended to develop the well-posedness for variational inequalities and their related problems, such as equilibrium problems, fixed point problems, inclusion problems and others; see e.g., [3, 11, 14, 17, 20].

It seems to be natural and easy to generalize the concept of well-posedness to hemivariational inequalities and to many people that most results on well-posedness for variational inequalities should hold for hemivariational inequalities under some similar conditions. However, it is not the truth. The Clarke's generalized directional derivative of a nonconvex and nonsmooth Lipschitz functional in hemivariational inequalities makes it much difficult. Thus, the literature on well-posedness for hemivariational inequalities is limit. In 1995, Goeleven and Mentagui [13] first introduced the well-posedness for a hemivariational inequality and presented some basic results concerning the well-posed hemivariational inequality. Later, using the concept of approximating sequence, Xiao et al. [34,36] defined a concept of well-posedness for a hemivariational inequality and a variational-hemivariational inequality. They gave some metric characterizations for the well-posed hemivariational inequality and the well-posed variational-hemivariational inequality, and proved the equivalence of well-posedness between the hemivariational inequality and the corresponding inclusion problem. Till now, there are a few papers discussing the solvability of systems of hemivariational inequalities since, due to the complex structure of systems of hemivariational inequalities, it is much more difficult than the study of hemivariational inequalities. Very recently, Wang et al. [32] introduced and considered the well-posedness for systems of hemivariational inequalities, gave some metric characterizations of well-posedness and established the equivalence between well-posedness of a system of hemivariational inequalities and its derived system of inclusion problems.

Inspired by the above research work of [32], in this paper we generalize the notion of $\alpha$-well-posedness to a system of time-dependent hemivariational inequalities without Volterra integral terms, establish some metric characterizations of $\alpha$-well-posedness and prove the equivalence between $\alpha$-well-posedness of the 
system of time-dependent hemivariational inequalities and its derived system of inclusion problems. The paper is structured as follows. In Section 2, we briefly recall some preliminaries. In Section 3, we define several notions of $\alpha$-well-posedness for the system of time-dependent hemivariational inequalities and, under some mild conditions on the operators involved, give some metric characterizations of $\alpha$-wellposedness for the system of time-dependent hemivariational inequalities. In Section 4, we establish two equivalence results of $\alpha$-well-posedness between the system of time-dependent hemivariational inequalities and its derived system of inclusion problems. Finally, in Section 5, we give some concluding remarks on our main results.

\section{Preliminaries}

In this section, we present the problem considered in this paper and recall some important notions and useful results on nonlinear analysis, nonsmooth analysis and operators of monotone type, which can be found in $[9,24,26,40]$.

Let $V_{1}$ and $V_{2}$ be real, separable and reflexive Banach spaces with dual spaces $V_{1}^{*}$ and $V_{2}^{*}$, respectively. Assume that $X_{1}$ and $X_{2}$ are real reflexive Banach spaces with dual spaces $X_{1}^{*}$ and $X_{2}^{*}$, respectively. For some $\mathrm{T} \in(0,+\infty)$, we suppose that $V_{1}=\mathrm{L}^{2}\left(0, \mathrm{~T} ; \mathrm{V}_{1}\right)$ and $V_{2}=\mathrm{L}^{2}\left(0, \mathrm{~T} ; \mathrm{V}_{2}\right)$, and thus $V_{1}^{*}=\mathrm{L}^{2}\left(0, \mathrm{~T} ; \mathrm{V}_{1}^{*}\right)$ and $V_{2}^{*}=\mathrm{L}^{2}\left(0, \mathrm{~T} ; \mathrm{V}_{2}^{*}\right)$. Unless otherwise specifically stated in the paper, we always denote by $\langle\cdot, \cdot\rangle_{\mathrm{E}^{*} \times \mathrm{E}}$ the duality pairing between Banach space $E$ and its dual $E^{*}$, and by $\|\cdot\|_{E},\|\cdot\|_{E^{*}}$ the norms on the space $E$ and its dual $E^{*}$, respectively, where $E \in\left\{V_{i}, X_{i}, V_{i}, i=1,2\right\}$. In this paper, we consider the following system of time-dependent hemivariational inequalities without Volterra integral terms, which is specified as follows: Find $\left(\mathfrak{u}_{1}, u_{2}\right) \in \mathcal{V}_{1} \times \mathcal{V}_{2}$ such that

$$
(\text { STDHVI })\left\{\begin{array}{c}
\left\langle A_{1}\left(t, u_{1}(t)\right)+B_{1}\left(t, u_{2}(t)\right), v_{1}\right\rangle_{V_{1}^{*} \times V_{1}}+J_{1}^{\circ}\left(t, M_{1}\left(u_{1}(t)\right), M_{2}\left(u_{2}(t)\right) ; M_{1} v_{1}\right) \\
\geqslant\left\langle f_{1}(t), v_{1}\right\rangle_{1}^{*} \times V_{1}, \quad \forall v_{1} \in V_{1}, \quad \text { a.e. } t \in(0, T), \\
\left\langle A_{2}\left(t, u_{2}(t)\right)+B_{2}\left(t, u_{1}(t)\right), v_{2}\right\rangle_{V_{2}^{*} \times V_{2}}+J_{2}^{\circ}\left(t, M_{1}\left(u_{1}(t)\right), M_{2}\left(u_{2}(t)\right) ; M_{2} v_{2}\right) \\
\geqslant\left\langle f_{2}(t), v_{2}\right\rangle_{V_{2}^{*} \times V_{2}}, \quad \forall v_{2} \in V_{2}, \quad \text { a.e. } t \in(0, T),
\end{array}\right.
$$

where, for $i, j=1,2$ and $j \neq i, A_{i}:(0, T) \times V_{i} \rightarrow V_{i}^{*}, B_{i}:(0, T) \times V_{j} \rightarrow V_{i}^{*}$, and $f_{i}:(0, T) \rightarrow V_{i}^{*}$ are operators with images in $V_{i}^{*}$. For $i \neq j=1,2, M_{i}: V_{i} \rightarrow X_{i}$ is a linear continuous and compact operator, and $J_{i}^{\circ}\left(t, x_{1}, x_{2} ; v\right)$ is the partial generalized directional derivative (in the sense of Clarke) of the locally Lipschitz functional $\mathrm{J}:(0, T) \times X_{1} \times X_{2} \rightarrow \mathbf{R}$ with respect to the $i$-th argument at the point $x_{i} \in X_{i}$ in the direction $v \in X_{i}$ for the given $x_{j} \in X_{j}$.

Suppose $Z$ is a real Banach space with its dual $Z^{*}$ and $\langle\cdot, \cdot\rangle_{Z^{*} \times Z}$ is the duality pairing between $Z^{*}$ and $Z$. Let $u$ and $\left\{u_{n}\right\}$ be a point and a sequence in $Z$, and let $u^{*}$ and $\left\{u_{n}^{*}\right\}$ be a point and a sequence in $Z^{*}$, respectively. We use the notations $u_{n} \rightarrow u, u_{n} \rightarrow u$ and $u_{n}^{*} \stackrel{*}{\rightarrow} u^{*}$ to indicate the strong convergence of $\left\{u_{n}\right\}$ to $u$, the weak convergence of $\left\{u_{n}\right\}$ to $u$ and the weak* convergence of $\left\{u_{n}^{*}\right\}$ to $u^{*}$, respectively. Recall that if $Z$ is not reflexive, then the weak* topology of $Z^{*}$ is weaker than its weak topology and that if $Z$ is reflexive, then the weak* topology of $Z^{*}$ coincides with its weak topology. It is easy to see that if $\left\{u_{n}\right\} \subset Z,\left\{u_{n}^{*}\right\} \subset V^{*}, u_{n} \rightarrow u$ in $Z$ and $u_{n}^{*} \stackrel{*}{\rightarrow} u^{*}$ in $Z^{*}$, then $\left\langle u_{n}^{*}, u_{n}\right\rangle Z^{*} \times Z \rightarrow\left\langle u^{*}, u\right\rangle_{Z^{*} \times Z}$ as $n \rightarrow \infty$.

Definition 2.1. Let $A: Z \rightarrow Z^{*}$ be an operator. $A$ is said to be

(i) demicontinuous, if for any sequence $\left\{u_{n}\right\} \subset Z$ with $u_{n} \rightarrow u \in Z, A\left(u_{n}\right) \rightarrow A(u)$ in $Z^{*}$;

(ii) hemicontinuous, if for all $u, v \in Z$, the function $t \mapsto\langle A(u+t(v-u)), v-u\rangle_{Z^{*} \times Z}$ from $[0,1]$ into $\mathbf{R}$ is continuous at $0^{+}$;

(iii) continuous, if for any sequence $\left\{u_{n}\right\} \subset Z$ with $u_{n} \rightarrow u \in Z, A\left(u_{n}\right) \rightarrow A(u)$ in $Z^{*}$.

It is clear that, if $A: Z \rightarrow Z^{*}$ is continuous, then it is demicontinuous which, in turn, implies that $A$ is hemicontinuous. If $A: Z \rightarrow Z^{*}$ is linear and demicontinuous, then it is continuous. It can be shown that for monotone operator $A: Z \rightarrow Z^{*}$ with $D(A)=Z$, the notions of demicontinuity and hemicontinuity coincide. 
Definition 2.2. An operator $A: Z \rightarrow Z^{*}$ is said to be monotone if

$$
\langle A u-A v, u-v\rangle_{Z^{*} \times Z} \geqslant 0, \quad \forall u, v \in Z .
$$

Definition 2.3. Let $h: Z \rightarrow R$ be a functional. $h$ is said to be

(i) Lipschitz continuous, if there exists a constant $\mathrm{L}>0$ such that

$$
\left|h\left(u_{1}\right)-h\left(u_{2}\right)\right| \leqslant L\left\|u_{1}-u_{2}\right\| z, \quad \forall u_{1}, u_{2} \in Z ;
$$

(ii) locally Lipschitz continuous, if for every $u \in Z$, there exist a neighborhood $N(u)$ and a constant $\mathrm{L}_{\mathfrak{u}}>0$ such that

$$
\left|h\left(u_{1}\right)-h\left(u_{2}\right)\right| \leqslant L_{u}\left\|u_{1}-u_{2}\right\|_{z}, \quad \forall u_{1}, u_{2} \in N(u) .
$$

Definition 2.4. Let $Z_{1}, Z_{2}$ be two real Banach spaces and $h: Z_{1} \times Z_{2} \rightarrow R$ be a functional. The functional $h$ is said to be

(i) Lipschitz continuous in the first variable if the functional $h\left(\cdot, u_{2}\right): Z_{1} \rightarrow Z_{1}^{*}$ is Lipschitz continuous on $Z_{1}$ for any given $u_{2} \in Z_{2}$;

(ii) locally Lipschitz continuous in the first variable, if the functional $h\left(\cdot, u_{2}\right): Z_{1} \rightarrow Z_{1}^{*}$ is locally Lipschitz continuous on $Z_{1}$ for any given $u_{2} \in Z_{2}$.

By the similar way, we can define the Lipschitz continuity and locally Lipschitz continuity of the functional $h: Z_{1} \times Z_{2} \rightarrow R$ in the second variable.

Definition 2.5. Let $h: Z \rightarrow \mathbf{R}$ be a locally Lipschitz functional on $Z$ and let $u, v \in Z$ be two given elements. The Clarke's generalized directional derivative of $h$ at the point $u$ in the direction $v$, denoted by $h^{\circ}(u ; v)$, is defined by

$$
h^{\circ}(u ; v)=\limsup _{w \rightarrow u, \lambda \downarrow 0} \frac{h(w+\lambda v)-h(w)}{\lambda} .
$$

The Clarke's generalized gradient of $h$ at $u$, denoted by $\partial h(u)$, the subset of the dual $Z^{*}$, which is defined by

$$
\partial h(u)=\left\{\rho \in Z^{*}: h^{\circ}(u ; v) \geqslant\langle\rho, v\rangle_{Z^{*} \times Z}, \forall v \in Z\right\} .
$$

Remark 2.6. As pointed out in [1], if $h: Z \rightarrow \mathbf{R}$ is convex and continuous, then the Clarke's generalized gradient $\partial h(x)$ coincides with the subdifferential of $h$ at $x$ in the sense of convex analysis, and if $h$ is continuously differentiable, then, for all $x \in Z, \partial h(x)=\left\{h^{\prime}(x)\right\}$, where $h^{\prime}(x)$ denotes the Fréchet differential of $h$ at $x$.

Proposition 2.7. Let $\mathrm{h}: \mathrm{Z} \rightarrow \mathbf{R}$ be a locally Lipschitz functional on $\mathrm{Z}$ and let $\mathrm{u}, \boldsymbol{v} \in \mathrm{Z}$ be two given elements. Then

(i) the function $v \mapsto \mathrm{h}^{\circ}(\mathrm{u} ; v)$ is finite, positively homogeneous, subadditive and thus convex on $\mathrm{Z}$;

(ii) $h^{\circ}(u ; v)$ is upper semicontinuous on $Z \times Z$ as a function of $(u, v)$, i.e., for all $u, v \in Z,\left\{u_{n}\right\} \subset Z,\left\{v_{n}\right\} \subset Z$ such that $\mathrm{u}_{\mathrm{n}} \rightarrow \mathrm{u}$ and $v_{\mathrm{n}} \rightarrow v$ in $\mathrm{Z}$, we have

$$
\limsup _{n \rightarrow \infty} h^{\circ}\left(u_{n} ; v_{n}\right) \leqslant h^{\circ}(u ; v) ;
$$

(iii) $\mathrm{h}^{\circ}(\mathrm{u} ;-\mathrm{v})=(-\mathrm{h})^{\circ}(\mathrm{u} ; \mathrm{v})$;

(iv) for all $\mathrm{u} \in \mathrm{Z}, \partial \mathrm{h}(\mathrm{u})$ is a nonempty, convex, bounded and weak*-compact subset of $\mathrm{Z}^{*}$;

(v) for all $v \in Z$, one has

$$
h^{\circ}(u ; v)=\max \left\{\langle\xi, v\rangle_{Z^{*} \times \mathbf{Z}}: \xi \in \partial \mathrm{h}(u)\right\} ;
$$

(vi) the graph of the Clarke's generalized gradient $\partial \mathrm{h}(\mathrm{u})$ is closed in $\mathrm{Z} \times\left(w^{*}-Z^{*}\right)$ topology, where $\left(w^{*}-Z^{*}\right)$ denotes the space $Z^{*}$ equipped with weak topology, i.e., if $\left\{u_{n}\right\} \subset Z$ and $\left\{u_{n}^{*}\right\} \subset Z^{*}$ are sequences such that $u_{n}^{*} \in \partial h\left(u_{n}\right), u_{n} \rightarrow u$ in $Z$ and $u_{n}^{*} \rightarrow u^{*}$ weakly* in $Z^{*}$, then $u^{*} \in \partial h(u)$. 
Definition 2.8. Let $h: Z \rightarrow \mathbf{R}$ be a locally Lipschitz functional on Banach space $Z$. $h$ is said to be regular (in the sense of Clarke) at $u \in Z$ if

(i) for all $v \in Z$, the directional derivative $h^{\prime}(u, v)$ exists;

(ii) for all $v \in Z, h^{\prime}(u, v)=h^{\circ}(u ; v)$, where $h^{\prime}(u, v)$ is the directional derivative of $h$ at $u \in Z$ in the direction $v \in Z$.

Proposition 2.9. Let $\mathrm{Z}_{1}$ and $\mathrm{Z}_{2}$ be two Banach spaces. If $\mathrm{H}: \mathrm{Z}_{1} \times \mathrm{Z}_{2} \rightarrow \mathbf{R}$ is locally Lipschitz on $\mathrm{Z}_{1} \times \mathrm{Z}_{2}$ and either $\mathrm{H}$ or $-\mathrm{H}$ is regular at $\left(\mathrm{u}_{1}, \mathrm{u}_{2}\right) \in \mathrm{Z}_{1} \times \mathrm{Z}_{2}$, then $\partial \mathrm{H}\left(\mathrm{u}_{1}, \mathrm{u}_{2}\right) \subseteq \partial_{1} \mathrm{H}\left(\mathrm{u}_{1}, \mathrm{u}_{2}\right) \times \partial_{2} \mathrm{H}\left(\mathrm{u}_{1}, \mathrm{u}_{2}\right)$, or for any point $\left(v_{1}, v_{2}\right) \in \mathrm{Z}_{1} \times \mathrm{Z}_{2}, \mathrm{H}^{\circ}\left(\mathrm{u}_{1}, \mathrm{u}_{2} ; v_{1}, v_{2}\right) \leqslant \mathrm{H}_{1}^{\circ}\left(\mathrm{u}_{1}, \mathrm{u}_{2} ; v_{1}\right)+\mathrm{H}_{2}^{\circ}\left(\mathrm{u}_{1}, \mathrm{u}_{2} ; v_{2}\right)$. However, in general, the converses of the above inclusion and inequality are not true.

Definition 2.10 (see [16]). Let $S$ be a nonempty subset of $Z$. The measure of noncompactness $\mu$ of the set $S$ is defined by

$$
\mu(S)=\inf \left\{\epsilon>0: S \subseteq \bigcup_{i=1}^{n} S_{i}, \operatorname{diam}\left(S_{i}\right)<\epsilon, i=1,2, \cdots, n\right\},
$$

where diam $\left(S_{i}\right)$ denotes the diameter of set $S_{i}$.

Definition 2.11. Let $A_{1}, A_{2}$ be nonempty subsets of $Z$. The Hausdorff metric $\mathcal{H}(\cdot, \cdot)$ between $A_{1}$ and $A_{2}$ is defined by

$$
\mathcal{H}\left(A_{1}, A_{2}\right)=\max \left\{e\left(A_{1}, A_{2}\right), e\left(A_{2}, A_{1}\right)\right\},
$$

where $e\left(A_{1}, A_{2}\right)=\sup _{a \in A_{1}} d\left(a, A_{2}\right)$ with $d\left(a, A_{2}\right)=\inf _{b \in A_{2}}\|a-b\|_{z}$.

Note that, in [16], we can find some more properties of the Hausdorff metric between two sets. At the end of this section, we give a lemma, which is important to our main results.

Theorem 2.12 (see [24]). Let $\mathrm{C} \subseteq \mathrm{Z}$ be nonempty, closed and convex, $\mathrm{C}^{*} \subseteq \mathrm{Z}^{*}$ be nonempty, closed, convex and bounded, $\varphi: Z \rightarrow \mathbf{R}$ be proper, convex and lower semicontinuous and $\mathrm{y} \in \mathrm{C}$ be arbitrary. Assume that, for each $x \in \mathrm{C}$, there exists $\chi^{*}(x) \in \mathrm{C}^{*}$ such that

$$
\left\langle x^{*}(x), x-y\right\rangle_{Z^{*} \times z} \geqslant \varphi(y)-\varphi(x) .
$$

Then, there exists $\mathrm{y}^{*} \in \mathrm{C}^{*}$ such that

$$
\left\langle y^{*}, x-y\right\rangle_{Z^{*} \times z} \geqslant \varphi(y)-\varphi(x), \quad \forall x \in C .
$$

\section{Well-posedness of STDHVI with metric characterizations}

In this section, based on the concepts of well-posedness for a system of hemivariational inequalities SHVI in [32], and $\alpha$-well-posedness for a system of mixed quasivariational-like inequalities in [4], we introduce the concept of $\alpha$-well-posedness for a system of time-dependent hemivariational inequalities STDHVI, and establish some metric characterizations of $\alpha$-well-posedness for STDHVI under some appropriate conditions.

Let $V=V_{1} \times V_{2}$. Endowed with the norm defined by $\|\mathbf{u}\|_{V}:=\left\|u_{1}\right\|_{v_{1}}+\left\|u_{2}\right\| V_{2}$ for all $\mathbf{u}=\left(u_{1}, u_{2}\right) \in V$, $\mathrm{V}$ is a reflexive Banach space with its dual $\mathrm{V}^{*}$ and the duality pairing between $\mathrm{V}$ and $\mathrm{V}^{*}$ is given by

$$
\left\langle\mathbf{u}^{*}, \mathbf{u}\right\rangle_{V^{*} \times \mathrm{V}}=\left\langle\mathrm{u}_{1}^{*}, \mathrm{u}_{1}\right\rangle_{\mathrm{V}_{1}^{*} \times \mathrm{V}_{1}}+\left\langle\mathrm{u}_{2}^{*}, \mathrm{u}_{2}\right\rangle_{\mathrm{V}_{2}^{*} \times \mathrm{V}_{2}}, \quad \forall \mathbf{u}^{*}=\left(\mathrm{u}_{1}^{*}, \mathrm{u}_{2}^{*}\right) \in \mathrm{V}^{*}, \quad \mathbf{u}=\left(\mathrm{u}_{1}, \mathrm{u}_{2}\right) \in \mathrm{V} .
$$

Similarly, we can also construct the product spaces $\mathcal{V}=\mathcal{V}_{1} \times \mathcal{V}_{2}$ and $\mathrm{X}=\mathrm{X}_{1} \times \mathrm{X}_{2}$ and their dual $\mathcal{V}^{*}$ and $X^{*}$.

For $i=1,2$, let $\alpha_{i}:(0, T) \times V_{i} \rightarrow \mathbf{R}^{+}=[0, \infty)$ be a functional such that for a.e. $t \in(0, T), \alpha_{i}(t, \cdot)$ is convex, continuous and positively homogeneous, i.e., $\alpha_{i}\left(t, \lambda_{i} v_{i}\right)=\lambda_{i} \alpha_{i}\left(t, v_{i}\right)$ for all $v_{i} \in V_{i}$ and $\lambda_{i} \geqslant 0$. 
Definition 3.1. A sequence $\left\{\mathbf{u}^{n}\right\} \subset \mathcal{V}$ with $\mathbf{u}^{n}=\left(u_{1}^{n}, u_{2}^{n}\right)$ is said to be an $\alpha$-approximating sequence with $\alpha=\left(\alpha_{1}, \alpha_{2}\right)$ for STDHVI if there exists $\epsilon_{\mathrm{n}}>0$ with $\epsilon_{\mathrm{n}} \rightarrow 0$ as $\mathrm{n} \rightarrow \infty$ such that

$$
\left\{\begin{array}{l}
\left\langle A_{1}\left(t, u_{1}^{n}(t)\right)+B_{1}\left(t, u_{2}^{n}(t)\right)-f_{1}(t), v_{1}-u_{1}^{n}(t)\right\rangle_{V_{1}^{*} \times V_{1}}+J_{1}^{\circ}\left(t, M_{1}\left(u_{1}^{n}(t)\right), M_{2}\left(u_{2}^{n}(t)\right) ; M_{1}\left(v_{1}-u_{1}^{n}(t)\right)\right) \\
\geqslant-\epsilon_{n} \alpha_{1}\left(t, v_{1}-u_{1}^{n}(t)\right), \quad \forall v_{1} \in V_{1}, \quad \text { a.e. } t \in(0, T), \\
\left\langle A_{2}\left(t, u_{2}^{n}(t)\right)+B_{2}\left(t, u_{1}^{n}(t)\right)-f_{2}(t), v_{2}-u_{2}^{n}(t)\right\rangle_{V_{2}^{*} \times V_{2}}+J_{2}^{\circ}\left(t, M_{1}\left(u_{1}^{n}(t)\right), M_{2}\left(u_{2}^{n}(t)\right) ; M_{2}\left(v_{2}-u_{2}^{n}(t)\right)\right) \\
\geqslant-\epsilon_{n} \alpha_{2}\left(t, v_{2}-u_{2}^{n}(t)\right), \quad \forall v_{2} \in V_{2}, \quad \text { a.e. } \quad t \in(0, T) .
\end{array}\right.
$$

In particular, if for $i=1,2, \alpha_{i}\left(t, x_{i}-y_{i}\right)=\left\|x_{i}-y_{i}\right\| v_{i}$ for all $x_{i}, y_{i} \in V_{i}$, then $\left\{\mathbf{u}^{n}\right\}$ is said to be an approximating sequence for STDHVI.

Definition 3.2. The system STDHVI of time-dependent hemivariational inequalities is said to be strongly (resp., weakly) $\alpha$-well-posed with $\alpha=\left(\alpha_{1}, \alpha_{2}\right)$ if it has a unique solution $\mathbf{u}$ and every $\alpha$-approximating sequence $\left\{\mathbf{u}^{\mathfrak{n}}\right\}$ for STDHVI has the property that for a.e. $t \in(0, T), \mathbf{u}^{\mathrm{n}}(t) \rightarrow \mathbf{u}(t)\left(\operatorname{resp} ., \mathbf{u}^{\mathrm{n}}(t) \rightarrow \mathbf{u}(t)\right)$ in V. In particular, if for $i=1,2, \alpha_{i}\left(t, x_{i}-y_{i}\right)=\left\|x_{i}-y_{i}\right\|_{v_{i}}$ for all $x_{i}, y_{i} \in v_{i}$, then the system STDHVI of time-dependent hemivariational inequalities is said to be strongly (resp., weakly) well-posed.

Remark 3.3. It is clear that, the strong $\alpha$-well-posedness of STDHVI implies the weak $\alpha$-well-posedness of STDHVI, but the converse is not true in general.

Definition 3.4. The system STDHVI of time-dependent hemivariational inequalities is said to be strongly (resp., weakly) $\alpha$-well-posed in the generalized sense if the solution set $S$ of STDHVI is nonempty and, for every $\alpha$-approximating sequence $\left\{\mathbf{u}^{\mathfrak{n}}\right\}$, there always exists a subsequence $\left\{\mathbf{u}^{n_{k}}\right\}$ such that for a.e. $t \in(0, T)$, $\mathbf{u}^{n_{k}}(t) \rightarrow \mathbf{u}(t)\left(\right.$ resp., $\left.\mathbf{u}^{n_{k}}(t) \rightarrow \mathbf{u}(t)\right)$ in $V$ for some $\mathbf{u} \in S$. In particular, if for $i=1,2$,

$$
\alpha_{i}\left(t, x_{i}-y_{i}\right)=\left\|x_{i}-y_{i}\right\|_{v_{i}}, \quad \forall x_{i}, y_{i} \in v_{i},
$$

then the system STDHVI of time-dependent hemivariational inequalities is said to be strongly (resp., weakly) well-posed in the generalized sense.

Remark 3.5. Similarly, the strong $\alpha$-well-posedness in the generalized sense for STDHVI implies the weak $\alpha$-well-posedness in the generalized sense for STDHVI, but the converse is not true in general.

Remark 3.6. The notions of strong and weak $\alpha$-well-posedness of STDHVI introduced in this paper are quite different from Definitions 3.1-3.2 and 3.4 in Wang et al. [32].

In order to establish the metric characterizations for the $\alpha$-well-posedness of STDHVI, we first define two sets in $\mathcal{V}=\mathcal{V}_{1} \times \mathcal{V}_{2}$ for any $\epsilon>0$ as follows:

$\Omega_{\alpha, \epsilon}=\left\{\mathbf{u}=\left(u_{1}, u_{2}\right) \in \mathcal{V}:\right.$ the following hold for all $\mathbf{v}=\left(v_{1}, v_{2}\right) \in V$ and a.e. $t \in(0, T)$

$$
\begin{aligned}
& \left\langle A_{1}\left(t, u_{1}(t)\right)+B_{1}\left(t, u_{2}(t)\right)-f_{1}(t), v_{1}-u_{1}(t)\right\rangle_{V_{1}^{*} \times V_{1}} \\
& \quad+J_{1}^{\circ}\left(t, M_{1}\left(u_{1}(t)\right), M_{2}\left(u_{2}(t)\right) ; M_{1}\left(v_{1}-u_{1}(t)\right)\right) \geqslant-\epsilon \alpha_{1}\left(t, v_{1}-u_{1}(t)\right), \\
& \left\langle A_{2}\left(t, u_{2}(t)\right)+B_{2}\left(t, u_{1}(t)\right)-f_{2}(t), v_{2}-u_{2}(t)\right\rangle_{2} \times V_{2} \\
& \left.\quad+J_{2}^{\circ}\left(t, M_{1}\left(u_{1}(t)\right), M_{2}\left(u_{2}(t)\right) ; M_{2}\left(v_{2}-u_{2}(t)\right)\right) \geqslant-\epsilon \alpha_{2}\left(t, v_{2}-u_{2}(t)\right)\right\},
\end{aligned}
$$

and

$$
\begin{aligned}
& \Delta_{\alpha, \epsilon}=\left\{\mathbf{u}=\left(u_{1}, u_{2}\right) \in \mathcal{V}:\right. \\
& \qquad \begin{array}{r}
\left\langle A_{1}\left(t, v_{1}\right)+B_{1}\left(t, u_{2}(t)\right)-f_{1}(t), v_{1}-u_{1}(t)\right\rangle V_{1}^{*} \times V_{1} \\
+ \\
\quad J_{1}^{\circ}\left(t, M_{1}\left(u_{1}(t)\right), M_{2}\left(u_{2}(t)\right) ; M_{1}\left(v_{1}-u_{1}(t)\right)\right) \geqslant-\epsilon \alpha_{1}\left(t, v_{1}-u_{1}(t)\right), \\
\left\langle A_{2}\left(t, v_{2}\right)+B_{2}\left(t, u_{1}(t)\right)-f_{2}(t), v_{2}-u_{2}(t)\right\rangle_{2}^{*} \times V_{2} \\
+ \\
\left.\quad J_{2}^{\circ}\left(t, M_{1}\left(u_{1}(t)\right), M_{2}\left(u_{2}(t)\right) ; M_{2}\left(v_{2}-u_{2}(t)\right)\right) \geqslant-\epsilon \alpha_{2}\left(t, v_{2}-u_{2}(t)\right)\right\} .
\end{array}
\end{aligned}
$$


Moreover, we also define two multi-valued mappings $\Omega_{\alpha, \epsilon}(\cdot):(0, \mathrm{~T}) \rightarrow 2^{\mathrm{V}}$ and $\Delta_{\alpha, \epsilon}(\cdot):(0, \mathrm{~T}) \rightarrow 2^{\mathrm{V}}$ as follows:

$$
\Omega_{\alpha, \epsilon}(\mathrm{t}):=\left\{\mathbf{u}(\mathrm{t}): \mathbf{u} \in \Omega_{\alpha, \epsilon}\right\} \quad \text { and } \quad \Delta_{\alpha, \epsilon}(\mathrm{t}):=\left\{\mathbf{u}(\mathrm{t}): \mathbf{u} \in \Delta_{\alpha, \epsilon}\right\}, \quad \forall \mathrm{t} \in(0, \mathrm{~T}) .
$$

In order to prove some properties of the sets $\Omega_{\alpha, \epsilon}$ and $\Delta_{\alpha, \epsilon}$, we first give some hypotheses on the operators $A_{1}, A_{2}, B_{1}, B_{2}$ and $J$ in the STDHVI.

(HAB) For $i, j=1,2$ and $j \neq i, A_{i}\left(\cdot, v_{i}\right):(0, T) \rightarrow V_{i}^{*}$ and $B_{i}\left(\cdot, v_{j}\right):(0, T) \rightarrow V_{i}^{*}$ both are measurable on $(0, \mathrm{~T})$ for all $\mathbf{v}=\left(v_{1}, v_{2}\right) \in \mathrm{V}$, and the following hold:

(1) for a.e. $t \in(0, T), A_{1}(t, \cdot): V_{1} \rightarrow V_{1}^{*}$ is monotone;

(2) for a.e. $t \in(0, T), A_{2}(t, \cdot): V_{2} \rightarrow V_{2}^{*}$ is monotone;

(3) for a.e. $t \in(0, T), A_{1}(t, \cdot): V_{1} \rightarrow V_{1}^{*}$ is demicontinuous on $V_{1}$;

(4) for a.e. $t \in(0, T), A_{2}(t, \cdot): V_{2} \rightarrow V_{2}^{*}$ is demicontinuous on $V_{2}$.

(5) for a.e. $t \in(0, T), B_{1}(t, \cdot): V_{2} \rightarrow V_{1}^{*}$ is demicontinuous on $V_{2}$;

(6) for a.e. $t \in(0, T), B_{2}(t, \cdot): V_{1} \rightarrow V_{2}^{*}$ is demicontinuous on $V_{1}$.

(HJ) $\mathrm{J}\left(\cdot, x_{1}, x_{2}\right):(0, T) \rightarrow R$ is measurable on $(0, T)$ for all $\left(x_{1}, x_{2}\right) \in X=X_{1} \times X_{2}$, and the following hold:

(1) for a.e. $t \in(0, T), J(t, \cdot, \cdot): X_{1} \times X_{2} \rightarrow \mathbf{R}$ is locally Lipschitz w.r.t. the first variable and second variable on $\mathrm{X}_{1} \times \mathrm{X}_{2}$

(2) for a.e. $t \in(0, T), J\left(t, x_{1}, x_{2}\right)+J\left(t, y_{1}, y_{2}\right)=J\left(t, x_{1}, y_{2}\right)+J\left(t, y_{1}, x_{2}\right), \forall\left(x_{1}, x_{2}\right),\left(y_{1}, y_{2}\right) \in X_{1} \times X_{2}$.

Lemma 3.7 ([32, Lemma 3.6]). Suppose that the functional J : $(0, T) \times X_{1} \times X_{2} \rightarrow \mathbf{R}$ satisfies the hypothesis (HJ). Then, for any sequence $\mathbf{x}^{\mathrm{n}}=\left(\mathrm{x}_{1}^{\mathrm{n}}, \mathrm{x}_{2}^{\mathrm{n}}\right) \in \mathrm{X}_{1} \times \mathrm{X}_{2}$ converging strongly to $\mathbf{x}=\left(\mathrm{x}_{1}, \mathrm{x}_{2}\right) \in \mathrm{X}_{1} \times \mathrm{X}_{2}$ and $\mathrm{y}_{i}^{\mathrm{n}} \in \mathrm{X}_{i}$ converging strongly to $\mathrm{y}_{i} \in \mathrm{X}_{i}$, one has

$$
\limsup _{n \rightarrow \infty} J_{i}^{\circ}\left(t, x_{1}^{n}, x_{2}^{n} ; y_{i}^{n}\right) \leqslant J_{i}^{\circ}\left(t, x_{1}, x_{2} ; y_{i}\right), \quad \text { for a.e. } t \in(0, T) \text {, }
$$

where $i=1,2$.

Lemma 3.8. Suppose that for $i, j=1,2$ and $j \neq i, A_{i}\left(\cdot, v_{i}\right):(0, T) \rightarrow V_{i}^{*}$ and $B_{i}\left(\cdot, v_{j}\right):(0, T) \rightarrow V_{i}^{*}$ satisfy the hypotheses (1)-(4) in (HAB) and $\mathrm{J}:(0, T) \times \mathrm{V}_{1} \times \mathrm{V}_{2} \rightarrow \mathbf{R}$ satisfies the hypothesis $(\mathbf{H J})$. Then, $\Omega_{\alpha, \epsilon}=\Delta_{\alpha, \epsilon}$ for all $\epsilon>0$.

Proof. For a.e. $t \in(0, T)$, by the monotonicity of operators $A_{1}(t, \cdot): V_{1} \rightarrow V_{1}^{*}$ and $A_{2}(t, \cdot): V_{2} \rightarrow V_{2}^{*}$, we can readily see that $\Omega_{\alpha, \epsilon} \subseteq \Delta_{\alpha, \epsilon}$ for any $\epsilon>0$. Thus we only need to show $\Delta_{\alpha, \epsilon} \subseteq \Omega_{\alpha, \epsilon}$. Indeed, take an arbitrary $\mathbf{u}=\left(u_{1}, u_{2}\right) \in \Delta_{\alpha, \epsilon}$. Then the following hold for all $\mathbf{v}=\left(v_{1}, v_{2}\right) \in V$ and a.e. $t \in(0, T)$,

$$
\left\{\begin{array}{c}
\left\langle A_{1}\left(t, v_{1}\right)+B_{1}\left(t, u_{2}(t)\right)-f_{1}(t), v_{1}-u_{1}(t)\right\rangle_{1}^{*} \times V_{1} \\
\quad+J_{1}^{\circ}\left(t, M_{1}\left(u_{1}(t)\right), M_{2}\left(u_{2}(t)\right) ; M_{1}\left(v_{1}-u_{1}(t)\right)\right) \geqslant-\epsilon \alpha_{1}\left(t, v_{1}-u_{1}(t)\right), \\
\left\langle A_{2}\left(t, v_{2}\right)+B_{2}\left(t, u_{1}(t)\right)-f_{2}(t), v_{2}-u_{2}(t)\right\rangle_{2}^{*} \times V_{2} \\
\quad+J_{2}^{\circ}\left(t, M_{1}\left(u_{1}(t)\right), M_{2}\left(u_{2}(t)\right) ; M_{2}\left(v_{2}-u_{2}(t)\right)\right) \geqslant-\epsilon \alpha_{2}\left(t, v_{2}-u_{2}(t)\right) .
\end{array}\right.
$$

For any $\mathbf{w}=\left(w_{1}, w_{2}\right) \in V_{1} \times V_{2}, \lambda \in(0,1)$, and a.e. $t \in(0, T)$, by letting $v_{1}=u_{1}(t)+\lambda\left(w_{1}-u_{1}(t)\right)$ and $v_{2}=u_{2}(t)+\lambda\left(w_{2}-u_{2}(t)\right)$ in (3.1), we deduce from (3.1) and the positive homogeneousness of $\alpha_{1}(t, \cdot)$ and $\alpha_{2}(t, \cdot)$ that for a.e. $t \in(0, T)$

$$
\left\{\begin{array}{c}
\left\langle A_{1}\left(t, u_{1}(t)+\lambda\left(w_{1}-u_{1}(t)\right)\right)+B_{1}\left(t, u_{2}(t)\right)-f_{1}(t), \lambda\left(w_{1}-u_{1}(t)\right)\right\rangle_{1}^{*} \times V_{1} \\
+J_{1}^{\circ}\left(t, M_{1}\left(u_{1}(t)\right), M_{2}\left(u_{2}(t)\right) ; \lambda M_{1}\left(w_{1}-u_{1}(t)\right)\right) \geqslant-\epsilon \lambda \alpha_{1}\left(t, w_{1}-u_{1}(t)\right), \\
\left\langle A_{2}\left(t, u_{2}(t)+\lambda\left(w_{2}-u_{2}(t)\right)\right)+B_{2}\left(t, u_{1}(t)\right)-f_{2}(t), \lambda\left(w_{2}-u_{2}(t)\right)\right\rangle_{2}^{*} \times v_{2} \\
+J_{2}^{\circ}\left(t, M_{1}\left(u_{1}(t)\right), M_{2}\left(u_{2}(t)\right) ; \lambda M_{2}\left(w_{2}-u_{2}(t)\right)\right) \geqslant-\epsilon \lambda \alpha_{2}\left(t, w_{2}-u_{2}(t)\right) .
\end{array}\right.
$$


From property (i) of Proposition 2.7, we know that Clarke's generalized directional derivative is positively homogeneous w.r.t. its direction. So, it follows that

$$
\left\{\begin{array}{c}
\left\langle A_{1}\left(t, u_{1}(t)+\lambda\left(w_{1}-u_{1}(t)\right)\right)+B_{1}\left(t, u_{2}(t)\right)-f_{1}(t), w_{1}-u_{1}(t)\right\rangle_{V_{1}^{*} \times V_{1}} \\
\quad+J_{1}^{\circ}\left(t, M_{1}\left(u_{1}(t)\right), M_{2}\left(u_{2}(t)\right) ; M_{1}\left(w_{1}-u_{1}(t)\right)\right) \geqslant-\epsilon \alpha_{1}\left(t, w_{1}-u_{1}(t)\right) \\
\left\langle A_{2}\left(t, u_{2}(t)+\lambda\left(w_{2}-u_{2}(t)\right)\right)+B_{2}\left(t, u_{1}(t)\right)-f_{2}(t), w_{2}-u_{2}(t)\right\rangle_{V_{2}^{*} \times V_{2}} \\
\quad+J_{2}^{\circ}\left(t, M_{1}\left(u_{1}(t)\right), M_{2}\left(u_{2}(t)\right) ; M_{2}\left(w_{2}-u_{2}(t)\right)\right) \geqslant-\epsilon \alpha_{2}\left(t, w_{2}-u_{2}(t)\right)
\end{array}\right.
$$

It is easy to see from the hypotheses (3), (4) in (HAB) that for a.e. $t \in(0, T)$, operators $A_{1}(t, \cdot): V_{1} \rightarrow V_{1}^{*}$ and $A_{2}(t, \cdot): V_{2} \rightarrow V_{2}^{*}$ both are hemicontinuous. Thus, taking the limit as $\lambda \rightarrow 0^{+}$at both sides of two inequalities in (3.2) implies that for a.e. $t \in(0, T)$,

$$
\left\{\begin{array}{l}
\left\langle A_{1}\left(t, u_{1}(t)\right)+B_{1}\left(t, u_{2}(t)\right)-f_{1}(t), w_{1}-u_{1}(t)\right\rangle_{V_{1}^{*} \times V_{1}} \\
\quad+J_{1}^{\circ}\left(t, M_{1}\left(u_{1}(t)\right), M_{2}\left(u_{2}(t)\right) ; M_{1}\left(w_{1}-u_{1}(t)\right)\right) \geqslant-\epsilon \alpha_{1}\left(t, w_{1}-u_{1}(t)\right), \\
\left\langle A_{2}\left(t, u_{2}(t)\right)+B_{2}\left(t, u_{1}(t)\right)-f_{2}(t), w_{2}-u_{2}(t)\right\rangle_{V_{2}^{*} \times V_{2}} \\
\quad+J_{2}^{\circ}\left(t, M_{1}\left(u_{1}(t)\right), M_{2}\left(u_{2}(t)\right) ; M_{2}\left(w_{2}-u_{2}(t)\right)\right) \geqslant-\epsilon \alpha_{2}\left(t, w_{2}-u_{2}(t)\right),
\end{array}\right.
$$

which together with the arbitrariness of $\mathbf{w}=\left(w_{1}, w_{2}\right) \in V_{1} \times V_{2}$, implies that $\mathbf{u} \in \Omega_{\alpha, \epsilon}$, and hence $\Delta_{\alpha, \epsilon} \subseteq \Omega_{\alpha, \epsilon}$. This completes the proof.

Lemma 3.9. Let $\mathrm{B}_{1}:(0, \mathrm{~T}) \times \mathrm{V}_{2} \rightarrow \mathrm{V}_{1}^{*}$ satisfy the hypothesis (5) in (HAB), $\mathrm{B}_{2}:(0, \mathrm{~T}) \times \mathrm{V}_{1} \rightarrow \mathrm{V}_{2}^{*}$ satisfy the hypothesis (6) in (HAB), and $\mathrm{J}:(0, \mathrm{~T}) \times \mathrm{X}_{1} \times \mathrm{X}_{2} \rightarrow \mathbf{R}$ satisfy the hypothesis (HJ). Then, for any $\epsilon>0$ and a.e. $\mathrm{t} \in(0, \mathrm{~T}), \Delta_{\alpha, \epsilon}(\mathrm{t})$ is closed in $\mathrm{V}_{1} \times \mathrm{V}_{2}$.

Proof. We let $t \in(0, T)$ be fixed. Let $\mathbf{u}^{n}(t)=\left(u_{1}^{n}(t), u_{2}^{n}(t)\right) \in \Delta_{\alpha, \epsilon}(t)$ be a sequence converging strongly to $\mathbf{u}(t)=\left(u_{1}(t), u_{2}(t)\right)$ in $v_{1} \times V_{2}$. Then

$$
\left\{\begin{array}{l}
\left\langle A_{1}\left(t, v_{1}\right)+B_{1}\left(t, u_{2}^{n}(t)\right)-f_{1}(t), v_{1}-u_{1}^{n}(t)\right\rangle_{V_{1}^{*} \times V_{1}} \\
\quad+J_{1}^{\circ}\left(t, M_{1}\left(u_{1}^{n}(t)\right), M_{2}\left(u_{2}^{n}(t)\right) ; M_{1}\left(v_{1}-u_{1}^{n}(t)\right)\right) \geqslant-\epsilon \alpha_{1}\left(t, v_{1}-u_{1}^{n}(t)\right), \quad \forall v_{1} \in V_{1}, \\
\quad\left\langle A_{2}\left(t, v_{2}\right)+B_{2}\left(t, u_{1}^{n}(t)\right)-f_{2}(t), v_{2}-u_{2}^{n}(t)\right\rangle_{V_{2}^{*} \times V_{2}}
\end{array}\right.
$$

Note that by the hypotheses (5), (6) in (HAB), the mappings $B_{1}(t, \cdot): V_{2} \rightarrow V_{1}^{*}$ and $B_{2}(t, \cdot): V_{1} \rightarrow V_{2}^{*}$ both are demicontinuous. So, it follows that $B_{1}\left(t, u_{2}^{n}(t)\right) \rightarrow B_{1}\left(t, u_{2}(t)\right)$ and $B_{2}\left(t, u_{1}^{n}(t)\right) \rightarrow B_{2}\left(t, u_{1}(t)\right)$. Thus, we get

$$
\left\{\begin{aligned}
\lim _{n \rightarrow \infty} & \left\langle A_{1}\left(t, v_{1}\right)+B_{1}\left(t, u_{2}^{n}(t)\right)-f_{1}(t), v_{1}-u_{1}^{n}(t)\right\rangle_{V_{1}^{*} \times V_{1}} \\
& =\left\langle A_{1}\left(t, v_{1}\right)+B_{1}\left(t, u_{2}(t)\right)-f_{1}(t), v_{1}-u_{1}(t)\right\rangle_{V_{1}^{*} \times V_{1}} \\
\lim _{n \rightarrow \infty} & \left\langle A_{2}\left(t, v_{2}\right)+B_{2}\left(t, u_{1}^{n}(t)\right)-f_{2}(t), v_{2}-u_{2}^{n}(t)\right\rangle_{V_{2}^{*} \times V_{2}} \\
= & \left\langle A_{2}\left(t, v_{2}\right)+B_{2}\left(t, u_{1}(t)\right)-f_{2}(t), v_{2}-u_{2}(t)\right\rangle_{V_{2}^{*} \times V_{2}} .
\end{aligned}\right.
$$

Moreover, by the hypothesis (HJ) on the functional J, Lemma 3.7 implies that

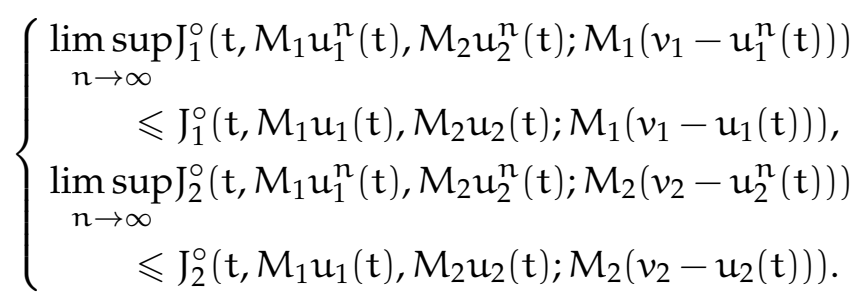


Also, from the continuity of $\alpha_{1}(t, \cdot)$ and $\alpha_{2}(t, \cdot)$ we get

$$
\lim _{n \rightarrow \infty} \alpha_{1}\left(t, v_{1}-u_{1}^{n}(t)\right)=\alpha_{1}\left(t, v_{1}-u_{1}(t)\right) \text { and } \lim _{n \rightarrow \infty} \alpha_{2}\left(t, v_{2}-u_{2}^{n}(t)\right)=\alpha_{2}\left(t, v_{2}-u_{2}(t)\right) .
$$

Therefore, taking the limsup as $n \rightarrow \infty$ at both sides of (3.3), we conclude from (3.4), (3.5) and (3.6) that

$$
\left\{\begin{array}{l}
\left\langle A_{1}\left(t, v_{1}\right)+B_{1}\left(t, u_{2}(t)\right)-f_{1}(t), v_{1}-u_{1}(t)\right\rangle_{V_{1}^{*} \times V_{1}} \\
\quad+J_{1}^{\circ}\left(t, M_{1}\left(u_{1}(t)\right), M_{2}\left(u_{2}(t)\right) ; M_{1}\left(v_{1}-u_{1}(t)\right)\right) \geqslant-\epsilon \alpha_{1}\left(t, v_{1}-u_{1}(t)\right), \quad \forall v_{1} \in V_{1}, \\
\left\langle A_{2}\left(t, v_{2}\right)+B_{2}\left(t, u_{1}(t)\right)-f_{2}(t), v_{2}-u_{2}(t)\right\rangle_{V_{2}^{*} \times V_{2}} \\
\quad+J_{2}^{\circ}\left(t, M_{1}\left(u_{1}(t)\right), M_{2}\left(u_{2}(t)\right) ; M_{2}\left(v_{2}-u_{2}(t)\right)\right) \geqslant-\epsilon \alpha_{2}\left(t, v_{2}-u_{2}(t)\right), \quad \forall v_{2} \in V_{2},
\end{array}\right.
$$

which implies that $\mathbf{u}(t)=\left(u_{1}(t), u_{2}(t)\right) \in \Delta_{\alpha, \epsilon}(t)$. Thus $\Delta_{\alpha, \epsilon}(t)$ is closed in $V_{1} \times V_{2}$. This completes the proof.

Remark 3.10. Let $A_{1}:(0, T) \times V_{1} \rightarrow V_{1}^{*}, A_{2}:(0, T) \times V_{2} \rightarrow V_{2}^{*}, B_{1}:(0, T) \times V_{2} \rightarrow V_{1}^{*}$ and $B_{2}:(0, T) \times V_{1} \rightarrow$ $\mathrm{V}_{2}^{*}$ satisfy the hypothesis (HAB) and $\mathrm{J}:(0, \mathrm{~T}) \times \mathrm{X}_{1} \times \mathrm{X}_{2} \rightarrow \mathbf{R}$ satisfy the hypothesis (HJ). Then it can be readily seen from Lemmas 3.8 and 3.9 that $\Omega_{\alpha, \epsilon}(t)=\Delta_{\alpha, \epsilon}(t)$ is closed in $V_{1} \times V_{2}$ for any $\epsilon>0$ and a.e. $t \in(0, T)$.

Theorem 3.11. Assume that there hold the hypothesis (3) on $A_{1}:(0, T) \times V_{1} \rightarrow V_{1}^{*}$, hypothesis (4) on $A_{2}:$ $(0, \mathrm{~T}) \times \mathrm{V}_{2} \rightarrow \mathrm{V}_{2}^{*}$, hypothesis (5) on $\mathrm{B}_{1}:(0, \mathrm{~T}) \times \mathrm{V}_{2} \rightarrow \mathrm{V}_{1}^{*}$ and hypothesis (6) on $\mathrm{A}_{2}:(0, \mathrm{~T}) \times \mathrm{V}_{1} \rightarrow \mathrm{V}_{2}^{*}$ in (HAB). Let $\mathrm{J}:(0, \mathrm{~T}) \times \mathrm{X}_{1} \times \mathrm{X}_{2} \rightarrow \mathbf{R}$ satisfy the hypothesis $(\mathbf{H J})$. Then the system STDHVI is strongly $\alpha$-well-posed if and only if for a.e. $\mathrm{t} \in(0, \mathrm{~T})$,

$$
\Omega_{\alpha, \epsilon}(\mathrm{t}) \neq \emptyset, \quad \forall \epsilon>0 \quad \text { and } \operatorname{diam}\left(\Omega_{\alpha, \epsilon}(\mathrm{t})\right) \rightarrow 0 \quad \text { as } \quad \epsilon \rightarrow 0 .
$$

Proof. Necessity. Let STDHVI be strongly $\alpha$-well-posed. Then STDHVI admits a unique solution $\mathbf{u}=\left(u_{1}, u_{2}\right) \in \mathcal{V}=\mathcal{V}_{1} \times \mathcal{V}_{2}$, i.e., for a.e. $t \in(0, T)$,

$$
\left\{\begin{array}{l}
\left\langle\mathrm{A}_{1}\left(\mathrm{t}, \mathrm{u}_{1}(\mathrm{t})\right)+\mathrm{B}_{1}\left(\mathrm{t}, \mathrm{u}_{2}(\mathrm{t})\right)-\mathrm{f}_{1}(\mathrm{t}), v_{1}-\mathrm{u}_{1}(\mathrm{t})\right\rangle_{\mathrm{V}_{1}^{*} \times \mathrm{V}_{1}} \\
\quad+\mathrm{J}_{1}^{\circ}\left(\mathrm{t}, \mathrm{M}_{1}\left(\mathrm{u}_{1}(\mathrm{t})\right), \mathrm{M}_{2}\left(\mathrm{u}_{2}(\mathrm{t})\right) ; \mathrm{M}_{1}\left(v_{1}-\mathrm{u}_{1}(\mathrm{t})\right)\right) \geqslant 0, \quad \forall v_{1} \in \mathrm{V}_{1}, \\
\left\langle\mathrm{~A}_{2}\left(\mathrm{t}, \mathrm{u}_{2}(\mathrm{t})\right)+\mathrm{B}_{2}\left(\mathrm{t}, \mathrm{u}_{1}(\mathrm{t})\right)-\mathrm{f}_{2}(\mathrm{t}), v_{2}-\mathrm{u}_{2}(\mathrm{t})\right\rangle_{\mathrm{V}_{2}^{*} \times \mathrm{V}_{2}} \\
\quad+\mathrm{J}_{2}^{\circ}\left(\mathrm{t}, \mathrm{M}_{1}\left(\mathrm{u}_{1}(\mathrm{t})\right), \mathrm{M}_{2}\left(\mathrm{u}_{2}(\mathrm{t})\right) ; \mathrm{M}_{2}\left(v_{2}-\mathrm{u}_{2}(\mathrm{t})\right)\right) \geqslant 0, \quad \forall v_{2} \in \mathrm{V}_{2}
\end{array}\right.
$$

This implies that $\mathbf{u}(t) \in \Omega_{\alpha, \epsilon}(t)$ for any $\epsilon>0$ and a.e. $t \in(0, T)$, i.e., $\Omega_{\alpha, \epsilon}(t) \neq \emptyset$ for all $\epsilon>0$ and a.e. $\mathrm{t} \in(0, \mathrm{~T})$. If for some $\mathrm{t}_{0} \in(0, \mathrm{~T}), \operatorname{diam}\left(\Omega_{\alpha, \epsilon}\left(\mathrm{t}_{0}\right)\right) \not \rightarrow 0$ as $\epsilon \rightarrow 0$, then there exist

$$
\mathbf{u}^{\mathfrak{n}}\left(t_{0}\right)=\left(u_{1}^{n}\left(t_{0}\right), u_{2}^{n}\left(t_{0}\right)\right) \in \Omega_{\alpha, \epsilon_{\mathfrak{n}}}\left(t_{0}\right), \quad \mathbf{p}^{\mathfrak{n}}\left(t_{0}\right)=\left(p_{1}^{\mathfrak{n}}\left(t_{0}\right), p_{2}^{\mathfrak{n}}\left(t_{0}\right)\right) \in \Omega_{\alpha, \epsilon_{\mathfrak{n}}}\left(t_{0}\right), \quad d>0,
$$

and $0<\epsilon_{\mathrm{n}} \rightarrow 0$ such that

$$
\left\|\mathbf{u}^{\mathrm{n}}\left(\mathrm{t}_{0}\right)-\mathbf{p}^{\mathrm{n}}\left(\mathrm{t}_{0}\right)\right\| \mathrm{v}_{1} \times \mathrm{v}_{2}=\left\|\mathrm{u}_{1}^{\mathrm{n}}\left(\mathrm{t}_{0}\right)-\mathrm{p}_{1}^{\mathrm{n}}\left(\mathrm{t}_{0}\right)\right\| \mathrm{v}_{1}+\left\|\mathrm{u}_{2}^{\mathrm{n}}\left(\mathrm{t}_{0}\right)-\mathrm{p}_{2}^{\mathrm{n}}\left(\mathrm{t}_{0}\right)\right\| \mathrm{v}_{2}>\mathrm{d} .
$$

Since the definition of $\Omega_{\alpha, \epsilon_{n}}\left(t_{0}\right)$ implies that $\mathbf{u}^{n} \in \Omega_{\alpha, \epsilon_{n}}$ and $\mathbf{p}^{n} \in \Omega_{\alpha, \epsilon_{n}}$, by the definition of the $\alpha$ approximating sequence for STDHVI, $\left\{\mathbf{u}^{\mathfrak{n}}\right\}$ and $\left\{\mathbf{p}^{\mathfrak{n}}\right\}$ are two $\alpha$-approximating sequences for STDHVI. Thus it follows from the strong $\alpha$-well-posedness of STDHVI that $\mathbf{u}^{\mathfrak{n}}\left(t_{0}\right) \rightarrow \mathbf{u}\left(t_{0}\right)$ and $\mathbf{p}^{\mathfrak{n}}\left(t_{0}\right) \rightarrow \mathbf{u}\left(t_{0}\right)$ in $\mathrm{V}$, which contradicts (3.7).

Sufficiency. Assume that for a.e. $t \in(0, T), \Omega_{\alpha, \epsilon}(t) \neq \emptyset$, for all $\epsilon>0$ and $\operatorname{diam}\left(\Omega_{\alpha, \epsilon}(t)\right) \rightarrow 0$ as $\epsilon \rightarrow 0$. Then we show that the system of time-dependent hemivariational inequalities STDHVI is strongly $\alpha$-wellposed. Indeed, suppose that $\left\{\mathbf{u}^{n}\right\}$ with $\mathbf{u}^{\mathfrak{n}}=\left(u_{1}^{n}, u_{2}^{n}\right)$ is an $\alpha$-approximating sequence for STDHVI. Then there exists $0<\epsilon_{n} \rightarrow 0$ as $n \rightarrow \infty$, such that

$$
\left\{\begin{array}{l}
\left\langle A_{1}\left(t, u_{1}^{n}(t)\right)+B_{1}\left(t, u_{2}^{n}(t)\right)-f_{1}(t), v_{1}-u_{1}^{n}(t)\right\rangle_{1}^{*} \times V_{1} \\
\quad+J_{1}^{\circ}\left(t, M_{1}\left(u_{1}^{n}(t)\right), M_{2}\left(u_{2}^{n}(t)\right) ; M_{1}\left(v_{1}-u_{1}^{n}(t)\right)\right) \geqslant-\epsilon_{n} \alpha_{1}\left(t, v_{1}-u_{1}^{n}(t)\right), \quad \forall v_{1} \in V_{1}, \text { a.e. } t \in(0, T), \\
\quad\left\langle A_{2}\left(t, u_{2}^{n}(t)\right)+B_{2}\left(t, u_{1}^{n}(t)\right)-f_{2}(t), v_{2}-u_{2}^{n}(t)\right\rangle_{2}^{*} \times V_{2} \\
\quad+J_{2}^{\circ}\left(t, M_{1}\left(u_{1}^{n}(t)\right), M_{2}\left(u_{2}^{n}(t)\right) ; M_{2}\left(v_{2}-u_{2}^{n}(t)\right)\right) \geqslant-\epsilon_{n} \alpha_{2}\left(t, v_{2}-u_{2}^{n}(t)\right), \quad \forall v_{2} \in V_{2}, \text { a.e. } t \in(0, T),
\end{array}\right.
$$


which implies $\mathbf{u}^{\mathrm{n}} \in \Omega_{\alpha, \epsilon_{n}}$ for all $\mathrm{n} \geqslant 1$. Since $\operatorname{diam}\left(\Omega_{\alpha, \epsilon_{n}}(\mathrm{t})\right) \rightarrow 0$ as $\mathrm{n} \rightarrow \infty$ for a.e. $\mathrm{t} \in(0, T),\left\{\mathbf{u}^{\mathrm{n}}(\mathrm{t})\right\}$ is a Cauchy sequence in $V$ for a.e. $t \in(0, T)$. Without loss of generality, we may assume that for a.e. $t \in(0, T)$, $\left\{\mathbf{u}^{\mathrm{n}}(\mathrm{t})\right\}$ converges strongly to $\mathbf{u}(\mathrm{t})=\left(\mathrm{u}_{1}(\mathrm{t}), \mathrm{u}_{2}(\mathrm{t})\right)$ in $\mathrm{V}=\mathrm{V}_{1} \times \mathrm{V}_{2}$.

Now, we claim that $\mathbf{u}$ is a unique solution to the system STDHVI of time-dependent hemivariational inequalities. Indeed, since operators $A_{1}(t, \cdot)+B_{1}(t, \cdot)$ and $A_{2}(t, \cdot)+B_{2}(t, \cdot)$ are demicontinuous on $V_{1} \times V_{2}$ (due to hypotheses (3)-(6) in (HAB)) and the functional J satisfies the hypothesis (HJ), we can obtain by the similar arguments to those in (3.4) and (3.5) that for a.e. $t \in(0, T)$,

$$
\begin{aligned}
\left\langle A_{1}\left(t, u_{1}(t)\right)+B_{1}\left(t, u_{2}(t)\right)-\right. & \left.f_{1}(t), v_{1}-u_{1}(t)\right\rangle_{V_{1}^{*} \times V_{1}}+J_{1}^{\circ}\left(t, M_{1}\left(u_{1}(t)\right), M_{2}\left(u_{2}(t)\right) ; M_{1}\left(v_{1}-u_{1}(t)\right)\right) \\
\geqslant & \lim _{n \rightarrow \infty}\left\langle A_{1}\left(t, u_{1}^{n}(t)\right)+B_{1}\left(t, u_{2}^{n}(t)\right)-f_{1}(t), v_{1}-u_{1}^{n}(t)\right\rangle_{V_{1}^{*} \times V_{1}} \\
& +\limsup _{n \rightarrow \infty}\left(t, M_{1}\left(u_{1}^{n}(t)\right), M_{2}\left(u_{2}^{n}(t)\right) ; M_{1}\left(v_{1}-u_{1}^{n}(t)\right)\right) \\
= & \limsup _{n \rightarrow \infty}\left\{\left\langle A_{1}\left(t, u_{1}^{n}(t)\right)+B_{1}\left(t, u_{2}^{n}(t)\right)-f_{1}(t), v_{1}-u_{1}^{n}(t)\right\rangle V_{1}^{*} \times V_{1}\right. \\
& \left.+J_{1}^{\circ}\left(t, M_{1}\left(u_{1}^{n}(t)\right), M_{2}\left(u_{2}^{n}(t)\right) ; M_{1}\left(v_{1}-u_{1}^{n}(t)\right)\right)\right\} \\
\geqslant & \lim _{n \rightarrow \infty}-\epsilon_{n} \alpha_{1}\left(t, v_{1}-u_{1}^{n}(t)\right) \\
= & 0 .
\end{aligned}
$$

By the similar way, one has for a.e. $t \in(0, T)$,

$$
\left\langle A_{2}\left(t, u_{2}(t)\right)+B_{2}\left(t, u_{1}(t)\right)-f_{2}(t), v_{2}-u_{2}(t)\right\rangle_{V_{2}^{*} \times V_{2}}+J_{2}^{\circ}\left(t, M_{1}\left(u_{1}(t)\right), M_{2}\left(u_{2}(t)\right) ; M_{2}\left(v_{2}-u_{2}(t)\right)\right) \geqslant 0 .
$$

Therefore, $\mathbf{u}$ is a solution to the system STDHVI.

Finally, we show the uniqueness of solutions of the system STDHVI. Suppose that $\mathbf{u}^{\prime}$ is another solution to the system STDHVI of time-dependent hemivariational inequalities. Since, for any $\epsilon>0$, $\mathbf{u}, \mathbf{u}^{\prime} \in \Omega_{\alpha, \epsilon}$,

$$
\left\|\mathbf{u}(\mathrm{t})-\mathbf{u}^{\prime}(\mathrm{t})\right\|_{\mathrm{V}_{1} \times \mathrm{V}_{2}} \leqslant \operatorname{diam}\left(\Omega_{\alpha, \epsilon}(\mathrm{t})\right),
$$

which together with the condition $\operatorname{diam}\left(\Omega_{\alpha, \epsilon}(\mathrm{t})\right) \rightarrow 0$ as $\epsilon \rightarrow 0$ for a.e. $t \in(0, T)$, implies that $\mathbf{u}=\mathbf{u}^{\prime}$. This completes the proof.

Theorem 3.12. Let $A_{1}:(0, T) \times V_{1} \rightarrow V_{1}^{*}, A_{2}:(0, T) \times V_{2} \rightarrow V_{2}^{*}, B_{1}:(0, T) \times V_{2} \rightarrow V_{1}^{*}$ and $B_{2}:(0, T) \times V_{1} \rightarrow$ $\mathrm{V}_{2}^{*}$ satisfy the hypothesis (HAB) and $\mathrm{J}:(0, \mathrm{~T}) \times \mathrm{X}_{1} \times \mathrm{X}_{2} \rightarrow \mathbf{R}$ satisfy the hypothesis $(\mathbf{H J})$. Then the system STDHVI is strongly $\alpha$-well-posed in the generalized sense if and only if for a.e. $t \in(0, T)$,

$$
\Omega_{\alpha, \epsilon}(\mathrm{t}) \neq \emptyset, \quad \forall \epsilon>0 \quad \text { and } \mu\left(\Omega_{\alpha, \epsilon}(\mathrm{t})\right) \rightarrow 0 \quad \text { as } \epsilon \rightarrow 0 .
$$

Proof. Necessity. Suppose that the system STDHVI of time-dependent hemivariational inequalities is strongly $\alpha$-well-posed in the generalized sense. Then the solution set of the system STDHVI, $S \neq \emptyset$. We define the multi-valued mapping $S(t):(0, T) \rightarrow 2^{\mathrm{V}}$ as follows:

$$
\mathrm{S}(\mathrm{t}):=\{\mathbf{u}(\mathrm{t}): \mathbf{u} \in \mathrm{S}\}, \quad \forall \mathrm{t} \in(0, \mathrm{~T}) .
$$

This means that, for any $\epsilon>0$ and a.e. $t \in(0, T), \Omega_{\alpha, \epsilon}(t) \neq \emptyset$ because $S(t) \subseteq \Omega_{\alpha, \epsilon}(t)$. Moreover, we claim here that the set $S(t)$ is compact in $V$ for a.e. $t \in(0, T)$. Indeed, for any sequence $\left\{\mathbf{u}^{\mathrm{n}}(t)\right\} \subset S(t)$ with $\mathbf{u}^{n}(t)=\left(u_{1}^{n}(t), u_{2}^{n}(t)\right)$, the corresponding $\left\{\mathbf{u}^{n}\right\} \subset S$ is an $\alpha$-approximating sequence for STDHVI and thus there exists a subsequence $\left\{\mathbf{u}^{n_{k}}\right\}$ such that for a.e. $t \in(0, T), \mathbf{u}^{n_{k}}(t) \rightarrow \mathbf{u}(t)$ in $V$ for some $\mathbf{u} \in S$, which implies that $S(t)$ is compact in $V$ for a.e. $t \in(0, T)$. To complete the proof of necessity, we show that $\mu\left(\Omega_{\alpha, \epsilon}(t)\right) \rightarrow 0$ as $\epsilon \rightarrow 0$ for a.e. $t \in(0, T)$. It follows from $S(t) \subseteq \Omega_{\alpha, \epsilon}(t)$ for a.e. $t \in(0, T)$ that

$$
\mathcal{H}\left(\Omega_{\alpha, \epsilon}(t), S(t)\right)=\max \left\{e\left(\Omega_{\alpha, \epsilon}(t), S(t)\right), e\left(S(t), \Omega_{\alpha, \epsilon}(t)\right)\right\}=e\left(\Omega_{\alpha, \epsilon}(t), S(t)\right) .
$$


Since the set $S(t)$ is compact for a.e. $t \in(0, T)$, one has for a.e. $t \in(0, T)$,

$$
\mu\left(\Omega_{\alpha, \epsilon}(t)\right) \leqslant 2 \mathcal{H}\left(\Omega_{\alpha, \epsilon}(t), S(t)\right)=2 e\left(\Omega_{\alpha, \epsilon}(t), S(t)\right) .
$$

Now, to show $\mu\left(\Omega_{\alpha, \epsilon}(t)\right) \rightarrow 0$ as $\epsilon \rightarrow 0$ for a.e. $t \in(0, T)$, it suffices to show that $e\left(\Omega_{\alpha, \epsilon}(t), S(t)\right) \rightarrow 0$ as $\epsilon \rightarrow 0$ for a.e. $t \in(0, T)$. Assume by contradiction that for some $t_{0} \in(0, T), e\left(\Omega_{\alpha, \epsilon}\left(t_{0}\right), S\left(t_{0}\right)\right) \not \rightarrow 0$ as $\epsilon \rightarrow 0$. Then there exist a constant $l>0$, a sequence $\left\{\epsilon_{n}\right\} \subset[0, \infty)$ with $\epsilon_{n} \rightarrow 0$ and $\mathbf{u}^{\mathrm{n}}\left(\mathrm{t}_{0}\right) \in \Omega_{\alpha, \epsilon_{\mathrm{n}}}\left(\mathrm{t}_{0}\right)$ such that

$$
\mathbf{u}^{\mathrm{n}}\left(\mathrm{t}_{0}\right) \notin \mathrm{S}\left(\mathrm{t}_{0}\right)+\mathrm{B}(0, \mathrm{l}),
$$

where $B(0, l)$ is the closed ball centered at 0 with radius $l$. Since $\mathbf{u}^{\mathrm{n}}\left(\mathrm{t}_{0}\right) \in \Omega_{\alpha, \epsilon_{\mathfrak{n}}}\left(\mathrm{t}_{0}\right)$ with $\epsilon_{\mathfrak{n}} \rightarrow 0$, the corresponding $\left\{\mathbf{u}^{\mathfrak{n}}\right\}$ is an $\alpha$-approximating sequence for STDHVI. So, there exists a subsequence $\left\{\mathbf{u}^{\mathfrak{n}_{k}}\right\}$ such that for a.e. $t \in(0, T), \mathbf{u}^{n_{k}}(t) \rightarrow \mathbf{u}(t)$ in $V$ for some $\mathbf{u} \in S$ (due to the strong $\alpha$-well-posedness in the generalized sense of STDHVI). This is a contradiction to (3.8). Consequently, $\mu\left(\Omega_{\alpha, \epsilon}(t)\right) \rightarrow 0$ as $\epsilon \rightarrow 0$ for a.e. $t \in(0, T)$.

Sufficiency. Assume that for a.e. $t \in(0, T), \Omega_{\alpha, \epsilon}(t) \neq \emptyset$, for all $\epsilon>0$ and $\mu\left(\Omega_{\alpha, \epsilon}(t)\right) \rightarrow 0$ as $\epsilon \rightarrow 0$. We show that the system STDHVI of time-dependent hemivariational inequalities is strongly $\alpha$-well-posed in the generalized sense. Indeed, we observe that for a.e. $t \in(0, T)$,

$$
S(t)=\bigcap_{\epsilon>0} \Omega_{\alpha, \epsilon}(t)
$$

Furthermore, since for a.e. $t \in(0, T), \mu\left(\Omega_{\alpha, \epsilon}(t)\right) \rightarrow 0$ as $\epsilon \rightarrow 0$ and by Remark 3.10, $\Omega_{\alpha, \epsilon}(t)$ is nonempty and closed for any $\epsilon>0$, it follows from the theorem in [10] that for a.e. $t \in(0, T), S(t)$ is nonempty compact and

$$
\left.e\left(\Omega_{\alpha, \epsilon}(t), S(t)\right)=\mathcal{H}\left(\Omega_{\alpha, \epsilon}(t)\right), S(t)\right) \rightarrow 0 \quad \text { as } \quad \epsilon \rightarrow 0 .
$$

Next, to show the strong $\alpha$-well-posedness in the generalized sense of STDHVI, let $\left\{\mathbf{u}^{n}\right\} \subset \mathcal{V}$ with $\mathbf{u}^{n}=\left(u_{1}^{n}, u_{2}^{n}\right)$ be an $\alpha$-approximating sequence for STDHVI. Then there exists a nonnegative sequence $\left\{\epsilon_{\mathrm{n}}\right\}$ with $\epsilon_{\mathrm{n}} \rightarrow 0$ such that

$$
\left\{\begin{array}{l}
\left\langle A_{1}\left(t, u_{1}^{n}(t)\right)+B_{1}\left(t, u_{2}^{n}(t)\right)-f_{1}(t), v_{1}-u_{1}^{n}(t)\right\rangle_{V_{1}^{*} \times V_{1}}+J_{1}^{o}\left(t, M_{1}\left(u_{1}^{n}(t)\right), M_{2}\left(u_{2}^{n}(t)\right) ; M_{1}\left(v_{1}-u_{1}^{n}(t)\right)\right) \\
\geqslant-\epsilon_{n} \alpha_{1}\left(t, v_{1}-u_{1}^{n}(t)\right), \quad \forall v_{1} \in V_{1}, \quad \text { a.e. } \quad t \in(0, T), \\
\left\langle A_{2}\left(t, u_{2}^{n}(t)\right)+B_{2}\left(t, u_{1}^{n}(t)\right)-f_{2}(t), v_{2}-u_{2}^{n}(t)\right\rangle_{V_{2}^{*} \times V_{2}}+J_{2}^{o}\left(t, M_{1}\left(u_{1}^{n}(t)\right), M_{2}\left(u_{2}^{n}(t)\right) ; M_{2}\left(v_{2}-u_{2}^{n}(t)\right)\right) \\
\geqslant-\epsilon_{n} \alpha_{2}\left(t, v_{2}-u_{2}^{n}(t)\right), \quad \forall v_{2} \in V_{2}, \quad \text { a.e. } \quad t \in(0, T),
\end{array}\right.
$$

which implies $\mathbf{u}^{\mathrm{n}}(\mathbf{t}) \in \Omega_{\alpha, \epsilon_{n}}(t)$. This together with (3.9), implies that for a.e. $t \in(0, T)$,

$$
d\left(\mathbf{u}^{n}(t), S(t)\right) \leqslant e\left(\Omega_{\alpha, \epsilon_{n}}(t), S(t)\right) \rightarrow 0 .
$$

We claim that there exists a subsequence $\left\{\mathbf{u}^{n_{k}}\right\}$ of $\left\{\mathbf{u}^{n}\right\}$ such that for a.e. $t \in(0, T), \mathbf{u}^{n_{k}}(t) \rightarrow \hat{\mathbf{u}}(t)$ in $V$ for some $\hat{\mathbf{u}} \in S$. Indeed, suppose that for every subsequence $\left\{\mathbf{u}^{n_{j}}\right\}$ of $\left\{\mathbf{u}^{\mathfrak{n}}\right\}$, there exists $t_{0} \in(0, T)$ such that $\mathbf{u}^{n_{j}}\left(t_{0}\right) \not \rightarrow u\left(t_{0}\right)$ in $V$ for all $\mathbf{u} \in S$. Then, from (3.10) and the compactness of $S\left(t_{0}\right)$, it follows that there exists $\overline{\mathbf{u}}^{\mathfrak{n}_{j}}\left(\mathrm{t}_{0}\right) \in \mathrm{S}\left(\mathrm{t}_{0}\right)$ such that as $\mathfrak{j} \rightarrow \infty$

$$
\left\|\mathbf{u}^{n_{j}}\left(t_{0}\right)-\overline{\mathbf{u}}^{n_{j}}\left(t_{0}\right)\right\|_{v_{1} \times V_{2}}=d\left(\mathbf{u}^{n_{j}}\left(t_{0}\right), S\left(t_{0}\right)\right) \leqslant e\left(\Omega_{\alpha, \epsilon_{n_{j}}}\left(t_{0}\right), S\left(t_{0}\right)\right) \rightarrow 0 .
$$

Again from the compactness of $S\left(t_{0}\right)$, we may, without loss of generality, that $\overline{\mathbf{u}}^{n_{j}}\left(t_{0}\right) \rightarrow \overline{\mathbf{u}}\left(t_{0}\right)$ in $V$ for some $\overline{\mathbf{u}} \in \mathrm{S}$. So, it follows that

$$
\left\|\mathbf{u}^{n_{j}}\left(t_{0}\right)-\overline{\mathbf{u}}\left(t_{0}\right)\right\| V_{1} \times V_{2} \leqslant\left\|\mathbf{u}^{n_{j}}\left(t_{0}\right)-\overline{\mathbf{u}}^{n_{j}}\left(t_{0}\right)\right\| V_{1} \times V_{2}+\left\|\overline{\mathbf{u}}^{n_{j}}\left(t_{0}\right)-\overline{\mathbf{u}}\left(t_{0}\right)\right\| V_{V_{1} \times V_{2}} \rightarrow 0,
$$

that is, $\mathbf{u}^{n_{\mathfrak{j}}}\left(t_{0}\right) \rightarrow \overline{\mathbf{u}}\left(t_{0}\right)$ in $V$ for some $\overline{\mathbf{u}} \in S$, which reaches a contradiction. Therefore, the system STDHVI is strongly $\alpha$-well-posed in the generalized sense. This completes the proof. 
Remark 3.13. The system SHVI of hemivariational inequalities considered in [32] is extended to develop the system STDHVI of time-dependent hemivariational inequalities without Volterra integral term. Our Lemmas 3.8-3.9 and Theorems 3.11-3.12 improve, extend and develop Lemmas 3.7-3.8 and Theorems 3.103.11 in [32] to a great extent because the system STDHVI of time-dependent hemivariational inequalities without Volterra integral term is very different from the system SHVI of hemivariational inequalities considered in [32, Lemmas 3.7-3.8 and Theorems 3.10-3.11].

\section{Relations with well-posedness of SIP}

In this section, we first introduce systems of inclusion problems in the product space $V_{1} \times V_{2}$ and then define the concept of $\alpha$-well-posedness for the system of inclusion problems. Moreover, we show the equivalence results between the $\alpha$-well-posedness of the system of time-dependent hemivariational inequalities and the $\alpha$-well-posedness of the corresponding system of inclusion problems.

Suppose that, for $i=1,2, \Gamma_{i}:(0, T) \times V_{1} \times V_{2} \rightarrow 2^{V_{i}^{*}}$ is a nonempty set-valued mapping. A system of inclusion problems associated with mappings $\Gamma_{1}$ and $\Gamma_{2}$ is defined as follows:

Find $\left(u_{1}, u_{2}\right) \in \mathcal{V}_{1} \times \mathcal{V}_{2}$ such that for a.e. $t \in(0, T)$,

$$
(\text { SIP }) \quad\left\{\begin{array}{l}
0_{1} \in \Gamma_{1}\left(t, u_{1}(t), u_{2}(t)\right), \\
0_{2} \in \Gamma_{2}\left(t, u_{1}(t), u_{2}(t)\right),
\end{array}\right.
$$

where for $i=1,2,0_{i} \in V_{i}^{*}$ represent the zero element in $V_{i}^{*}$. For simplicity, we use the symbols as follows:

$$
\mathbf{u}=\left(\mathrm{u}_{1}, \mathrm{u}_{2}\right) \in \mathcal{V}=\mathcal{V}_{1} \times \mathcal{V}_{2}, \quad \mathbf{0}=\left(0_{1}, 0_{2}\right) \in \mathrm{V}^{*}=\mathrm{V}_{1}^{*} \times \mathrm{V}_{2}^{*}, \quad \Gamma(\mathrm{t}, \mathbf{u}(\mathrm{t}))=\left(\Gamma_{1}(\mathrm{t}, \mathbf{u}(\mathrm{t})), \Gamma_{2}(\mathrm{t}, \mathbf{u}(\mathrm{t}))\right) .
$$

This allows us to simplify the system of inclusion problems as follows:

Find $\mathbf{u} \in \mathcal{V}$ such that for a.e. $t \in(0, T)$,

$$
\mathbf{0} \in \Gamma(t, \mathbf{u}(t))
$$

Definition 4.1. A sequence $\left\{\mathbf{u}^{n}\right\} \subset \mathcal{V}_{1} \times \mathcal{V}_{2}$ with $\mathbf{u}^{n}=\left(u_{1}^{n}, u_{2}^{n}\right)$ is called an $\alpha$-approximating sequence for the system SIP of inclusion problems if there exist $\mathbf{p}^{n}(t)=\left(p_{1}^{n}(t), p_{2}^{n}(t)\right) \in \Gamma\left(t, \mathbf{u}^{n}(t)\right), n \in \mathbf{N}$ for a.e. $t \in(0, T)$ and a nonnegative sequence $\left\{\epsilon_{n}\right\}$ with $\left\|\mathbf{p}^{n}(t)\right\| V_{1}^{*} \times V_{2}^{*}+\epsilon_{n} \rightarrow 0$ as $n \rightarrow \infty$ for a.e. $t \in(0, T)$, such that for a.e. $t \in(0, T)$,

$$
\left\{\begin{array}{lll}
\left\langle p_{1}^{n}(t), v_{1}-u_{1}^{n}(t)\right\rangle V_{1}^{*} \times V_{1} \geqslant-\epsilon_{n} \alpha_{1}\left(t, v_{1}-u_{1}^{n}(t)\right), & \forall v_{1} \in V_{1}, & n \in N \\
\left\langle p_{2}^{n}(t), v_{2}-u_{2}^{n}(t)\right\rangle_{2}^{*} \times V_{2} \geqslant-\epsilon_{n} \alpha_{2}\left(t, v_{2}-u_{2}^{n}(t)\right), & \forall v_{2} \in V_{2}, & n \in N
\end{array}\right.
$$

Definition 4.2. The system SIP is said to be strongly (resp., weakly) $\alpha$-well-posed if it has a unique solution $\mathbf{u}$ and every $\alpha$-approximating sequence $\left\{\mathbf{u}^{\mathrm{n}}\right\}$ has the property that for a.e. $t \in(0, T), \mathbf{u}^{\mathrm{n}}(\mathbf{t}) \rightarrow \mathbf{u}(t)$ (resp., $\left.\mathbf{u}^{\mathrm{n}}(\mathrm{t}) \rightarrow \mathbf{u}(\mathrm{t})\right)$ in $\mathrm{V}$.

Definition 4.3. The system SIP is said to be strongly (resp., weakly) $\alpha$-well-posed in the generalized sense if the solution set $S$ of SIP is nonempty and, for every $\alpha$-approximating sequence $\left\{\mathbf{u}^{n}\right\}$, there always exists a subsequence $\left\{\mathbf{u}^{n_{k}}\right\}$ such that for a.e. $t \in(0, T), \mathbf{u}^{n_{k}}(t) \rightarrow \mathbf{u}(t)\left(\right.$ resp., $\left.\mathbf{u}^{n_{k}}(t) \rightarrow \mathbf{u}(t)\right)$ in $V$ for some $\mathbf{u} \in S$.

For $\mathrm{t} \in(0, \mathrm{~T})$ and $\mathbf{v}=\left(v_{1}, v_{2}\right) \in \mathrm{V}$, we define some operators as follows:

$$
\left\{\begin{array}{l}
A:(0, T) \times V \rightarrow V^{*}, \quad A(t, v):=\left(A_{1}\left(t, v_{1}\right)+B_{1}\left(t, v_{2}\right), A_{2}\left(t, v_{2}\right)+B_{2}\left(t, v_{1}\right)\right), \\
M: V \rightarrow X=X_{1} \times X_{2}, \quad M v:=\left(M_{1} v_{1}, M_{2} v_{2}\right), \\
f:(0, T) \rightarrow V^{*}, \quad f(t):=\left(f_{1}(t), f_{2}(t)\right) .
\end{array}\right.
$$

In order to show that the $\alpha$-well-posedness of the system of time-dependent hemivariational inequalities is equivalent to the $\alpha$-well-posedness of its corresponding system of inclusion problems, we first give a lemma which establishes the equivalence between the system STDHVI and its derived system of inclusion problems. 
Lemma 4.4. Let locally Lipschitz functional $\mathrm{J}(\mathrm{t}, \cdot, \cdot)$ be regular on $\mathrm{X}$ for a.e. $\mathrm{t} \in(0, \mathrm{~T})$. Then, $\mathbf{u}=\left(\mathrm{u}_{1}, \mathrm{u}_{2}\right) \in$ $\mathcal{V}_{1} \times \mathcal{V}_{2}$ is a solution to the system STDHVI of time-dependent hemivariational inequalities if and only if it solves the following derived system of inclusion problems: Find $\mathbf{u}=\left(\mathrm{u}_{1}, \mathrm{u}_{2}\right) \in \mathcal{V}_{1} \times \mathcal{V}_{2}$ such that for a.e. $\mathrm{t} \in(0, \mathrm{~T})$,

$$
\text { (DSIP) }\left\{\begin{array}{l}
f_{1}(t) \in A_{1}\left(t, u_{1}(t)\right)+B_{1}\left(t, u_{2}(t)\right)+M_{1}^{*} \circ \partial_{1} J\left(t, M_{1} u_{1}(t), M_{2} u_{2}(t)\right), \\
f_{2}(t) \in A_{2}\left(t, u_{2}(t)\right)+B_{2}\left(t, u_{1}(t)\right)+M_{2}^{*} \circ \partial_{2} J\left(t, M_{1} u_{1}(t), M_{2} u_{2}(t)\right),
\end{array}\right.
$$

where for $i \neq j=1,2, M_{i}^{*}$ is the adjoint operator of $M_{i}, \partial_{i} J\left(t, x_{1}, x_{2}\right)$ denotes the Clarke's generalized gradient of the functional $\mathrm{J}\left(\mathrm{t}, \cdot, \mathrm{x}_{\mathrm{j}}\right)$ at $\mathrm{x}_{\mathrm{i}}$.

Proof. First of all, we show the necessity. Indeed, assume that $\mathbf{u}=\left(u_{1}, u_{2}\right) \in V_{1} \times \mathcal{V}_{2}$ is a solution to the system STDHVI of time-dependent hemivariational inequalities, i.e., for all $\mathbf{v}=\left(v_{1}, v_{2}\right) \in \mathrm{V}$ and a.e. $t \in(0, T)$,

$$
\left\{\begin{array}{l}
\left\langle A_{1}\left(t, u_{1}(t)\right)+B_{1}\left(t, u_{2}(t)\right)-f_{1}(t), v_{1}\right\rangle_{V_{1}^{*} \times V_{1}}+J_{1}^{\circ}\left(t, M_{1}\left(u_{1}(t)\right), M_{2}\left(u_{2}(t)\right) ; M_{1} v_{1}\right) \geqslant 0 \\
\left\langle A_{2}\left(t, u_{2}(t)\right)+B_{2}\left(t, u_{1}(t)\right)-f_{2}(t), v_{2}\right\rangle_{V_{2}^{*} \times v_{2}}+J_{2}^{\circ}\left(t, M_{1}\left(u_{1}(t)\right), M_{2}\left(u_{2}(t)\right) ; M_{2} v_{2}\right) \geqslant 0
\end{array}\right.
$$

which is hence equivalent to the following

$$
\left\{\begin{array}{l}
J_{1}^{\circ}\left(t, M_{1}\left(u_{1}(t)\right), M_{2}\left(u_{2}(t)\right) ; M_{1} v_{1}\right) \geqslant\left\langle f_{1}(t)-A_{1}\left(t, u_{1}(t)\right)-B_{1}\left(t, u_{2}(t)\right), v_{1}\right\rangle_{V_{1}^{*} \times V_{1}}, \\
J_{2}^{\circ}\left(t, M_{1}\left(u_{1}(t)\right), M_{2}\left(u_{2}(t)\right) ; M_{2} v_{2}\right) \geqslant\left\langle f_{2}(t)-A_{2}\left(t, u_{2}(t)\right)-B_{2}\left(t, u_{1}(t)\right), v_{2}\right\rangle_{V_{2}^{*} \times V_{2}} .
\end{array}\right.
$$

Moreover, since $J(t, \cdot, \cdot)$ is regular and $M_{i}$ is linear bounded for $i=1,2$, it follows from Proposition 3.37 in monograph [24] that $J_{1}^{\circ}\left(t, M_{1}\left(u_{1}(t)\right), M_{2}\left(u_{2}(t)\right) ; M_{1} v_{1}\right)=\left(J\left(t, \cdot, M_{2} u_{2}(t)\right) \circ M_{1}\right)^{\circ}\left(u_{1}(t) ; v_{1}\right)$ and $\partial\left(J\left(t, \cdot, M_{2} u_{2}(t)\right) \circ M_{1}\right)\left(u_{1}(t)\right)=M_{1}^{*} \circ \partial_{1} J\left(t, M_{1} u_{1}(t), M_{2} u_{2}(t)\right)$ for all $v_{1} \in V_{1}$ and a.e. $t \in(0, T)$ and that $J_{2}^{\circ}\left(t, M_{1}\left(u_{1}(t)\right), M_{2}\left(u_{2}(t)\right) ; M_{2} v_{2}\right)=\left(J\left(t, M_{1} u_{1}(t), \cdot\right) \circ M_{2}\right)^{\circ}\left(u_{2}(t) ; v_{2}\right)$ and

$$
\partial\left(J\left(t, M_{1} u_{1}(t), \cdot\right) \circ M_{2}\right)\left(u_{2}(t)\right)=M_{2}^{*} \circ \partial_{2} J\left(t, M_{1} u_{1}(t), M_{2} u_{2}(t)\right)
$$

for all $v_{2} \in V_{2}$ and a.e. $t \in(0, T)$. So, it follows from (4.1) that for a.e. $t \in(0, T)$,

$$
\left\{\begin{array}{l}
f_{1}(t) \in A_{1}\left(t, u_{1}(t)\right)+B_{1}\left(t, u_{2}(t)\right)+M_{1}^{*} \circ \partial_{1} J\left(t, M_{1} u_{1}(t), M_{2} u_{2}(t)\right), \\
f_{2}(t) \in A_{2}\left(t, u_{2}(t)\right)+B_{2}\left(t, u_{1}(t)\right)+M_{2}^{*} \circ \partial_{2} J\left(t, M_{1} u_{1}(t), M_{2} u_{2}(t)\right),
\end{array}\right.
$$

which implies that $\mathbf{u}=\left(u_{1}, u_{2}\right) \in \mathcal{V}_{1} \times \mathcal{V}_{2}$ is the solution to the system DSIP.

Sufficiency. Suppose that $\mathbf{u}=\left(\mathfrak{u}_{1}, \mathfrak{u}_{2}\right) \in \mathcal{V}_{1} \times \mathcal{V}_{2}$ is a solution to the derived system DSIP of inclusion problems, i.e., for a.e. $t \in(0, T)$,

$$
(\text { DSIP }) \quad\left\{\begin{array}{l}
f_{1}(t) \in A_{1}\left(t, u_{1}(t)\right)+B_{1}\left(t, u_{2}(t)\right)+M_{1}^{*} \circ \partial_{1} J\left(t, M_{1} u_{1}(t), M_{2} u_{2}(t)\right), \\
f_{2}(t) \in A_{2}\left(t, u_{2}(t)\right)+B_{2}\left(t, u_{1}(t)\right)+M_{2}^{*} \circ \partial_{2} J\left(t, M_{1} u_{1}(t), M_{2} u_{2}(t)\right) .
\end{array}\right.
$$

Then, there exist $\eta_{1}(t) \in \partial_{1} J\left(t, M_{1} u_{1}(t), M_{2} u_{2}(t)\right)$ and $\eta_{2}(t) \in \partial_{2} J\left(t, M_{1} u_{1}(t), M_{2} u_{2}(t)\right)$ such that for a.e. $t \in(0, T)$,

$$
f_{1}(t)=A_{1}\left(t, u_{1}(t)\right)+B_{1}\left(t, u_{2}(t)\right)+M_{1}^{*} \eta_{1}(t) \quad \text { and } \quad f_{2}(t)=A_{2}\left(t, u_{2}(t)\right)+B_{2}\left(t, u_{1}(t)\right)+M_{2}^{*} \eta_{2}(t) .
$$

By multiplying the above two equalities in (4.2) with $v_{1}$ and $v_{2}$, respectively, we can obtain by the definition of the Clarke's generalized gradient, that for all $\mathbf{v}=\left(v_{1}, v_{2}\right) \in V_{1} \times V_{2}$ and a.e. $t \in(0, T)$,

$$
\begin{aligned}
\left\langle f_{1}(t), v_{1}\right\rangle_{V_{1}^{*} \times v_{1}} & =\left\langle A_{1}\left(t, u_{1}(t)\right)+B_{1}\left(t, u_{2}(t)\right)+M_{1}^{*} \eta_{1}(t), v_{1}\right\rangle_{1}^{*} \times v_{1} \\
& =\left\langle A_{1}\left(t, u_{1}(t)\right)+B_{1}\left(t, u_{2}(t)\right), v_{1}\right\rangle V_{1}^{*} \times v_{1}+\left\langle\eta_{1}(t), M v_{1}\right\rangle v_{1}^{*} \times v_{1} \\
& \leqslant\left\langle A_{1}\left(t, u_{1}(t)\right)+B_{1}\left(t, u_{2}(t)\right), v_{1}\right\rangle v_{1}^{*} \times v_{1}+J_{1}^{\circ}\left(t, M_{1} u_{1}(t), M_{2} u_{2}(t) ; M_{1} v_{1}\right),
\end{aligned}
$$


and

$$
\begin{aligned}
\left\langle f_{2}(t), v_{2}\right\rangle_{V_{2}^{*} \times V_{2}} & =\left\langle A_{2}\left(t, u_{2}(t)\right)+B_{2}\left(t, u_{1}(t)\right)+M_{2}^{*} \eta_{2}(t), v_{2}\right\rangle_{V_{2}^{*} \times V_{2}} \\
& =\left\langle A_{2}\left(t, u_{2}(t)\right)+B_{2}\left(t, u_{1}(t)\right), v_{2}\right\rangle_{V_{2}^{*} \times v_{2}}+\left\langle\eta_{2}(t), M v_{2}\right\rangle V_{2}^{*} \times v_{2} \\
& \leqslant\left\langle A_{2}\left(t, u_{2}(t)\right)+B_{2}\left(t, u_{1}(t)\right), v_{2}\right\rangle_{2}^{*} \times v_{2}+J_{2}^{\circ}\left(t, M_{1} u_{1}(t), M_{2} u_{2}(t) ; M_{2} v_{2}\right) .
\end{aligned}
$$

Therefore, $\mathbf{u}$ is a solution of the system STDHVI. This completes the proof.

Remark 4.5. It is easy to simplify the system DSIP as follows: Find $\mathbf{u} \in \mathcal{V}$ such that for a.e. $t \in(0, T)$,

$$
\mathbf{0} \in A(t, \mathbf{u})-f(t)+\Theta(t, \mathbf{u}),
$$

where $\Theta(t, \mathbf{v}):=\left(M_{1}^{*} \circ \partial_{1} J\left(t, M_{1} v_{1}, M_{2} v_{2}\right), M_{2}^{*} \circ \partial_{2} J\left(t, M_{1} v_{1}, M_{2} v_{2}\right)\right)$ for all $\mathbf{v}=\left(v_{1}, v_{2}\right) \in V$ and $t \in(0, T)$.

Theorem 4.6. Let locally Lipschitz functional $\mathrm{J}(\mathrm{t}, \cdot, \cdot)$ be regular on $\mathrm{X}$ for a.e. $\mathrm{t} \in(0, \mathrm{~T})$. Then, the system STDHVI of time-dependent hemivariational inequalities is strongly $\alpha$-well-posed if and only if the derived system DSIP of inclusion problems is strongly $\alpha$-well-posed.

Proof. Necessity. Suppose that the system STDHVI of time-dependent hemivariational inequalities is strongly $\alpha$-well-posed. Then there exists a unique $\mathbf{u}=\left(\mathfrak{u}_{1}, \mathfrak{u}_{2}\right) \in \mathcal{V}_{1} \times \mathcal{V}_{2}$ solving STDHVI. It follows from Lemma 4.4 that $\mathbf{u}$ is the unique solution to DSIP. To show the strong $\alpha$-well-posedness of DSIP, we let $\mathbf{u}^{n}=\left(u_{1}^{n}, u_{2}^{n}\right)$ be an $\alpha$-approximating sequence for DSIP. We claim that for a.e. $t \in(0, T), \mathbf{u}^{n}(t) \rightarrow \mathbf{u}(t)$ in $V$. Indeed, one knows that there exist $\mathbf{p}^{n}(t)=\left(p_{1}^{n}(t), p_{2}^{n}(t)\right) \in A(t, \mathbf{u}(t))-f(t)+\Theta\left(t, \mathbf{u}^{n}(t)\right), n \in N$ for a.e. $t \in(0, T)$ and a nonnegative sequence $\left\{\epsilon_{n}\right\}$ with $\left\|p^{n}(t)\right\|_{V_{1}^{*} \times V_{2}^{*}}+\epsilon_{n} \rightarrow 0$ as $n \rightarrow \infty$ for a.e. $t \in(0, T)$, such that for a.e. $t \in(0, T)$,

$$
\left\{\begin{array}{lll}
\left\langle p_{1}^{\mathfrak{n}}(t), v_{1}-u_{1}^{\mathfrak{n}}(t)\right\rangle_{V_{1}^{*} \times V_{1} \geqslant-\epsilon_{n}} \alpha_{1}\left(t, v_{1}-u_{1}^{n}(t)\right), & \forall v_{1} \in V_{1}, & n \in N \\
\left\langle p_{2}^{n}(t), v_{2}-u_{2}^{n}(t)\right\rangle_{2}^{*} \times V_{2} \geqslant-\epsilon_{n} \alpha_{2}\left(t, v_{2}-u_{2}^{n}(t)\right), & \forall v_{2} \in V_{2}, & n \in N
\end{array}\right.
$$

It is clear that for a.e. $t \in(0, T)$,

$$
\left\{\begin{array}{l}
p_{1}^{n}(t)-A_{1}\left(t, u_{1}^{n}(t)\right)-B_{1}\left(t, u_{2}^{n}(t)\right)+f_{1}(t) \in M_{1}^{*} \circ \partial_{1} J\left(t, M_{1} u_{1}^{n}(t), M_{2} u_{2}^{n}(t)\right), \\
p_{2}^{n}(t)-A_{2}\left(t, u_{2}^{n}(t)\right)-B_{2}\left(t, u_{1}^{n}(t)\right)+f_{2}(t) \in M_{2}^{*} \circ \partial_{2} J\left(t, M_{1} u_{1}^{n}(t), M_{2} u_{2}^{n}(t)\right) .
\end{array}\right.
$$

By the similar arguments to those in the proof of Lemma 4.4, one easily obtains that for all $\mathbf{v}=\left(v_{1}, v_{2}\right) \in \mathrm{V}$ and a.e. $t \in(0, T)$,

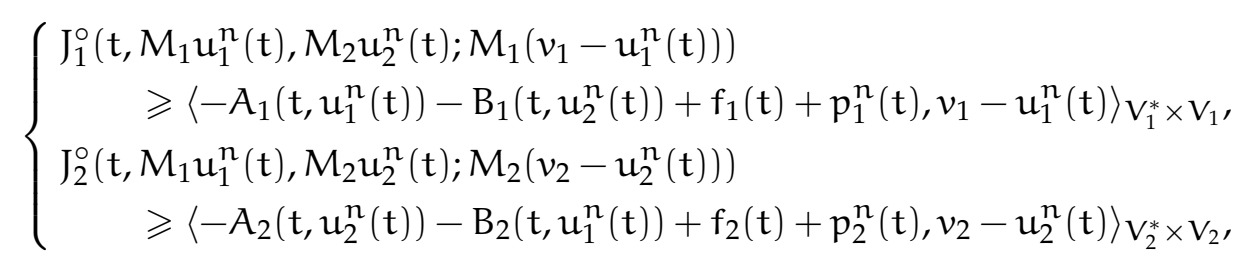

which together with (4.3), implies that for all $\mathbf{v}=\left(v_{1}, v_{2}\right) \in \mathrm{V}$ and a.e. $t \in(0, T)$,

$$
\left\{\begin{array}{l}
\left\langle A_{1}\left(t, u_{1}^{n}(t)\right)+B_{1}\left(t, u_{2}^{n}(t)\right)-f_{1}(t), v_{1}-u_{1}^{n}(t)\right\rangle_{V_{1}^{*} \times V_{1}}+J_{1}^{\circ}\left(t, M_{1} u_{1}^{n}(t), M_{2} u_{2}^{n}(t) ; M_{1}\left(v_{1}-u_{1}^{n}(t)\right)\right) \\
\quad \geqslant\left\langle p_{1}^{n}(t), v_{1}-u_{1}^{n}(t)\right\rangle_{1}^{*} \times V_{1} \geqslant-\epsilon_{n} \alpha_{1}\left(t, v_{1}-u_{1}^{n}(t)\right), \\
\left\langle A_{2}\left(t, u_{2}^{n}(t)\right)+B_{2}\left(t, u_{1}^{n}(t)\right)-f_{2}(t), v_{2}-u_{2}^{n}(t)\right\rangle_{V_{2}^{*} \times V_{2}}+J_{2}^{\circ}\left(t, M_{1} u_{1}^{n}(t), M_{2} u_{2}^{n}(t) ; M_{2}\left(v_{2}-u_{2}^{n}(t)\right)\right) \\
\quad \geqslant\left\langle p_{2}^{n}(t), v_{2}-u_{2}^{n}(t)\right\rangle_{V_{2}^{*} \times V_{2}} \geqslant-\epsilon_{n} \alpha_{2}\left(t, v_{2}-u_{2}^{n}(t)\right) .
\end{array}\right.
$$

This means that $\left\{\mathbf{u}^{n}\right\}$ is an $\alpha$-approximating sequence for STDHVI. Therefore, it follows from the strong $\alpha$-well-posedness of STDHVI that for a.e. $t \in(0, T), \mathbf{u}^{n}(t) \rightarrow \mathbf{u}(t)$ in V. Consequently, DISP is strongly $\alpha$-well-posed. 
Sufficiency. Let the system DSIP of inclusion problems be strongly $\alpha$-well-posed. Then there exists a unique solution $\mathbf{u}$ to DSIP, which together with Lemma 4.4, implies that $\mathbf{u}$ is the unique solution to the system STDHVI of time-dependent hemivariational inequalities as well. Let $\left\{\mathbf{u}^{\mathfrak{n}}\right\}$ be an $\alpha$-approximating sequence for STDHVI. Then there exists a nonnegative sequence $\left\{\epsilon_{n}\right\}$ with $\epsilon_{n} \rightarrow 0$ as $n \rightarrow \infty$ such that

$$
\left\{\begin{array}{c}
\left\langle A_{1}\left(t, u_{1}^{n}(t)\right)+B_{1}\left(t, u_{2}^{n}(t)\right)-f_{1}(t), v_{1}-u_{1}^{n}(t)\right\rangle_{V_{1}^{*} \times V_{1}} \\
\quad+J_{1}^{\circ}\left(t, M_{1}\left(u_{1}^{n}(t)\right), M_{2}\left(u_{2}^{n}(t)\right) ; M_{1}\left(v_{1}-u_{1}^{n}(t)\right)\right) \\
\geqslant-\epsilon_{n} \alpha_{1}\left(t, v_{1}-u_{1}^{n}(t)\right), \quad \forall v_{1} \in V_{1}, \quad \text { a.e. } \quad t \in(0, T), \\
\left\langle A_{2}\left(t, u_{2}^{n}(t)\right)+B_{2}\left(t, u_{1}^{n}(t)\right)-f_{2}(t), v_{2}-u_{2}^{n}(t)\right\rangle_{V_{2}^{*} \times V_{2}} \\
\quad+J_{2}^{\circ}\left(t, M_{1}\left(u_{1}^{n}(t)\right), M_{2}\left(u_{2}^{n}(t)\right) ; M_{2}\left(v_{2}-u_{2}^{n}(t)\right)\right) \\
\quad \geqslant-\epsilon_{n} \alpha_{2}\left(t, v_{2}-u_{2}^{n}(t)\right), \quad \forall v_{2} \in V_{2}, \quad \text { a.e. } \quad t \in(0, T) .
\end{array}\right.
$$

By virtue of Proposition $2.7(v)$, one observes that for all $\mathbf{v}=\left(v_{1}, v_{2}\right) \in V$ and a.e. $t \in(0, T)$,

$$
\left\{\begin{array}{l}
J_{1}^{\circ}\left(t, M_{1} u_{1}^{n}(t), M_{2} u_{2}^{n}(t) ; M_{1}\left(v_{1}-u_{1}^{n}(t)\right)\right) \\
\quad=\max \left\{\left\langle h_{1}, M_{1}\left(v_{1}-u_{1}^{n}(t)\right)\right\rangle_{V_{1}^{*} \times V_{1}}: h_{1} \in \partial_{1} J\left(t, M_{1} u_{1}^{n}(t), M_{2} u_{2}^{n}(t)\right)\right\}, \\
\quad J_{2}^{\circ}\left(t, M_{1} u_{1}^{n}(t), M_{2} u_{2}^{n}(t) ; M_{2}\left(v_{2}-u_{2}^{n}(t)\right)\right) \\
\quad=\max \left\{\left\langle h_{2}, M_{2}\left(v_{2}-u_{2}^{n}(t)\right)\right\rangle_{V_{2}^{*} \times V_{2}}: h_{2} \in \partial_{2} J\left(t, M_{1} u_{1}^{n}(t), M_{2} u_{2}^{n}(t)\right)\right\}
\end{array}\right.
$$

Thus, for any $\mathbf{v}=\left(v_{1}, v_{2}\right) \in V$ and a.e. $t \in(0, T)$, there exist

$$
h_{1}\left(t, u_{1}^{n}(t), u_{2}^{n}(t), v_{1}\right) \in \partial_{1} J\left(t, M_{1} u_{1}^{n}(t), M_{2} u_{2}^{n}(t)\right) \text { and } h_{2}\left(t, u_{1}^{n}(t), u_{2}^{n}(t), v_{2}\right) \in \partial_{2} J\left(t, M_{1} u_{1}^{n}(t), M_{2} u_{2}^{n}(t)\right)
$$

such that

$$
\left\{\begin{array}{l}
\left\langle A_{1}\left(t, u_{1}^{n}(t)\right)+B_{1}\left(t, u_{2}^{n}(t)\right)-f_{1}(t), v_{1}-u_{1}^{n}(t)\right\rangle_{V_{1}^{*} \times V_{1}} \\
\quad+\left\langle h_{1}\left(t, u_{1}^{n}(t), u_{2}^{n}(t), v_{1}\right), M_{1}\left(v_{1}-u_{1}^{n}(t)\right)\right\rangle_{1}^{*} \times V_{1} \geqslant-\epsilon_{n} \alpha_{1}\left(t, v_{1}-u_{1}^{n}(t)\right), \\
\left\langle A_{2}\left(t, u_{2}^{n}(t)\right)+B_{2}\left(t, u_{1}^{n}(t)\right)-f_{2}(t), v_{2}-u_{2}^{n}(t)\right\rangle_{V_{2}^{*} \times V_{2}} \\
\quad+\left\langle h_{2}\left(t, u_{1}^{n}(t), u_{2}^{n}(t), v_{2}\right), M_{2}\left(v_{2}-u_{2}^{n}(t)\right)\right\rangle_{V_{2}^{*} \times V_{2}} \geqslant-\epsilon_{n} \alpha_{2}\left(t, v_{2}-u_{2}^{n}(t)\right) .
\end{array}\right.
$$

According to Proposition 2.7 (iv), one knows that for a.e. $t \in(0, T)$, both $\partial_{1} J\left(t, M_{1} u_{1}^{n}(t), M_{2} u_{2}^{n}(t)\right)$ and $\partial_{2} J\left(t, M_{1} u_{1}^{n}(t), M_{2} u_{2}^{n}(t)\right)$ are nonempty, convex, bounded and closed in $V_{1}^{*}$ and $V_{2}^{*}$, respectively. So, it follows that for a.e. $t \in(0, T)$, the sets $\left\{A_{1}\left(t, u_{1}^{n}(t)\right)+B_{1}\left(t, u_{2}^{n}(t)\right)+p_{1}-f_{1}(t): p_{1} \in \partial_{1} J\left(t, M_{1} u_{1}^{n}(t), M_{2} u_{2}^{n}(t)\right)\right\}$ and $\left\{A_{2}\left(t, u_{2}^{n}(t)\right)+B_{2}\left(t, u_{1}^{n}(t)\right)+p_{2}-f_{2}(t): p_{2} \in \partial_{2} J\left(t, M_{1} u_{1}^{n}(t), M_{2} u_{2}^{n}(t)\right)\right\}$ are also nonempty, convex, bounded and closed in $V_{1}^{*}$ and $V_{2}^{*}$, respectively. Therefore, for $i=1,2$, it follows from (4.4) and Theorem 2.12 with $\varphi_{i}(t, \cdot)=\epsilon_{n} \alpha_{i}\left(t, \cdot-u_{i}^{n}(t)\right)$ that for a.e. $t \in(0, T)$, there exists

$$
\mathbf{h}^{\mathrm{n}}(\mathrm{t})=\left(\mathrm{h}_{1}^{\mathrm{n}}(\mathrm{t}), \mathrm{h}_{2}^{\mathrm{n}}(\mathrm{t})\right) \in \partial_{1} J\left(t, M_{1} u_{1}^{n}(t), M_{2} u_{2}^{n}(t)\right) \times \partial_{2} J\left(t, M_{1} u_{1}^{n}(t), M_{2} u_{2}^{n}(t)\right),
$$

which is independent from $\mathbf{v}=\left(v_{1}, v_{2}\right)$, such that for all $\mathbf{v}=\left(v_{1}, v_{2}\right) \in \mathrm{V}$ and a.e. $\mathrm{t} \in(0, \mathrm{~T})$,

$$
\left\{\begin{array}{c}
\left\langle A_{1}\left(t, u_{1}^{n}(t)\right)+B_{1}\left(t, u_{2}^{n}(t)\right)-f_{1}(t), v_{1}-u_{1}^{n}(t)\right\rangle_{V_{1}^{*} \times V_{1}} \\
\quad+\left\langle h_{1}^{n}(t), M_{1}\left(v_{1}-u_{1}^{n}(t)\right)\right\rangle_{V_{1}^{*} \times V_{1}} \geqslant-\epsilon_{n} \alpha_{1}\left(t, v_{1}-u_{1}^{n}(t)\right) \\
\left\langle A_{2}\left(t, u_{2}^{n}(t)\right)+B_{2}\left(t, u_{1}^{n}(t)\right)-f_{2}(t), v_{2}-u_{2}^{n}(t)\right\rangle_{V_{2}^{*} \times V_{2}} \\
\quad+\left\langle h_{2}^{n}(t), M_{2}\left(v_{2}-u_{2}^{n}(t)\right)\right\rangle_{2}^{*} \times V_{2} \geqslant-\epsilon_{n} \alpha_{2}\left(t, v_{2}-u_{2}^{n}(t)\right)
\end{array}\right.
$$

So, it follows from (4.5) that for a.e. $t \in(0, T)$,

$$
\left\{\begin{array}{lll}
\left\langle p_{1}^{n}(t), v_{1}-u_{1}^{n}(t)\right\rangle_{V_{1}^{*} \times V_{1} \geqslant-\epsilon_{n}} \alpha_{1}\left(t, v_{1}-u_{1}^{n}(t)\right), & \forall v_{1} \in V_{1}, & n \in N, \\
\left\langle p_{2}^{n}(t), v_{2}-u_{2}^{n}(t)\right\rangle_{V_{2}^{*} \times V_{2}} \geqslant-\epsilon_{n} \alpha_{2}\left(t, v_{2}-u_{2}^{n}(t)\right), & \forall v_{2} \in V_{2}, & n \in N,
\end{array}\right.
$$


where

$$
p_{1}^{n}(t)=A_{1}\left(t, u_{1}^{n}(t)\right)+B_{1}\left(t, u_{2}^{n}(t)\right)-f_{1}(t)+M_{1}^{*} h_{1}^{n}(t)
$$

and

$$
p_{2}^{n}(t)=A_{2}\left(t, u_{2}^{n}(t)\right)+B_{2}\left(t, u_{1}^{n}(t)\right)-f_{2}(t)+M_{2}^{*} h_{2}^{n}(t) .
$$

It is clear that for a.e. $t \in(0, T)$,

$$
\begin{cases}p_{1}^{n}(t) \in A_{1}\left(t, u_{1}^{n}(t)\right)+B_{1}\left(t, u_{2}^{n}(t)\right)-f_{1}(t)+M_{1}^{*} \circ \partial_{1} J\left(t, M_{1} u_{1}^{n}(t), M_{2} u_{2}^{n}(t)\right), & \forall n \in N \\ p_{2}^{n}(t) \in A_{2}\left(t, u_{2}^{n}(t)\right)+B_{2}\left(t, u_{1}^{n}(t)\right)-f_{2}(t)+M_{2}^{*} \circ \partial_{2} J\left(t, M_{1} u_{1}^{n}(t), M_{2} u_{2}^{n}(t)\right), & \forall n \in N\end{cases}
$$

That is, $\mathbf{p}^{\mathfrak{n}}(t)=\left(p_{1}^{\mathfrak{n}}(t), p_{2}^{\mathfrak{n}}(t)\right) \in A(t, \mathbf{u}(t))-f(t)+\Theta\left(t, \mathbf{u}^{n}(t)\right), n \in \mathbf{N}$ for a.e. $t \in(0, T)$.

Next, to show $\left\|\mathbf{p}^{\mathfrak{n}}(\mathrm{t})\right\|_{V^{*}} \rightarrow 0$ as $\mathrm{n} \rightarrow \infty$ for a.e. $t \in(0, T)$, it is sufficient to show $\left\|p_{i}^{n}(t)\right\|_{V_{i}^{*}} \rightarrow 0$ as $n \rightarrow \infty$ for a.e. $t \in(0, T)$ where $i=1$, 2. Indeed, let $t \in(0, T)$ be fixed. Then, $\left\|p_{i}^{n}(t)\right\|_{V_{i}^{*}} \rightarrow 0$ as $n \rightarrow \infty$ if and only if for any $\varepsilon>0$ there exists an integer $N \geqslant 1$ such that $\left\|p_{i}^{n}(t)\right\|_{V_{i}^{*}}<\varepsilon$ for all $n \geqslant N$. If for some $t_{0} \in(0, T),\left\|p_{i}^{n}\left(t_{0}\right)\right\| V_{i}^{*} \not \rightarrow 0$ as $n \rightarrow \infty$, then there exists $\varepsilon_{i}>0$ and for each $k \geqslant 1$ there exists $p_{i}^{n_{k}}\left(t_{0}\right)$ such that

$$
\left\|p_{i}^{n_{k}}\left(t_{0}\right)\right\|_{V_{i}^{*}} \geqslant \varepsilon_{i} .
$$

Note that $V_{i}$ is reflexive, i.e., $V_{i}=V_{i}^{* *}$. According to the normalized duality mapping $\partial_{i}$ from $V_{i}^{*}$ to its dual $\mathrm{V}_{i}^{* *}\left(=\mathrm{V}_{i}\right)$ defined by

$$
\partial_{i}\left(v_{i}\right)=\left\{x_{i} \in V_{i}:\left\langle v_{i}, x_{i}\right\rangle_{V_{i}^{*} \times V_{i}}=\left\|v_{i}\right\|_{V_{i}^{*}}^{2}=\left\|x_{i}\right\|_{V_{i}}^{2}\right\}, \quad \forall v_{i} \in V_{i}^{*},
$$

we know that for each $n \in \mathbf{N}$ there exists $j_{i}\left(p_{i}^{n}\left(t_{0}\right)\right) \in \mathcal{J}_{i}\left(p_{i}^{n}\left(t_{0}\right)\right)$ such that

$$
\left\langle p_{i}^{n}\left(t_{0}\right), j_{i}\left(p_{i}^{n}\left(t_{0}\right)\right)\right\rangle_{V_{i}^{*} \times V_{i}}=\left\|p_{i}^{n}\left(t_{0}\right)\right\|_{V_{i}^{*}}^{2}=\left\|j_{i}\left(p_{i}^{n}\left(t_{0}\right)\right)\right\|_{V_{i}}^{2}
$$

Putting $\mathrm{t}=\mathrm{t}_{0}$ and $v_{i}=u_{i}^{n}\left(t_{0}\right)-j_{i}\left(p_{i}^{n}\left(t_{0}\right)\right)$ in (4.6), we get

$$
\left\langle p_{i}^{n}\left(t_{0}\right),-j_{i}\left(p_{i}^{n}\left(t_{0}\right)\right)\right\rangle_{V_{i}^{*} \times V_{i}} \geqslant-\epsilon_{n} \alpha_{i}\left(t_{0},-j_{i}\left(p_{i}^{n}\left(t_{0}\right)\right)\right), \quad \forall n \in N,
$$

which immediately implies that

$$
-\left\|p_{i}^{n}\left(t_{0}\right)\right\|_{V_{i}^{*}}^{2} \geqslant-\epsilon_{n} \alpha_{i}\left(t_{0},-j_{i}\left(p_{i}^{n}\left(t_{0}\right)\right)\right), \quad \forall n \in \mathbf{N} .
$$

Taking into account $\left\|\frac{j_{i}\left(p_{i}^{n_{k}}\left(t_{0}\right)\right)}{\left\|p_{i}^{n_{k}}\left(t_{0}\right)\right\|_{v_{i}^{*}}}\right\| v_{i}=1$ for all $k \geqslant 1$, we obtain that $\left\|\epsilon_{n_{k}} \frac{j_{i}\left(p_{i}^{n_{k}}\left(t_{0}\right)\right)}{\left\|p_{i}^{n_{k}}\left(t_{0}\right)\right\|_{v_{i}^{*}}}\right\|_{v_{i}} \rightarrow 0$ as $k \rightarrow \infty$. Utilizing the continuity and positive homogeneity of $\alpha_{i}\left(t_{0}, \cdot\right)$, we deduce from (4.7) that

$$
\begin{aligned}
\varepsilon_{i} \leqslant\left\|p_{i}^{n_{k}}\left(t_{0}\right)\right\|_{V_{i}^{*}} & \leqslant \frac{\epsilon_{n_{k}}}{\left\|p_{i}^{n_{k}}\left(t_{0}\right)\right\| V_{i}^{*}} \alpha_{i}\left(t_{0},-j_{i}\left(p_{i}^{n_{k}}\left(t_{0}\right)\right)\right) \\
& =\alpha_{i}\left(t_{0},-\epsilon_{n_{k}} \frac{j_{i}\left(p_{i}^{n_{k}}\left(t_{0}\right)\right)}{\left\|p_{i}^{n_{k}}\left(t_{0}\right)\right\|_{V_{i}^{*}}}\right) \rightarrow 0 \text { as } k \rightarrow \infty,
\end{aligned}
$$

which reaches a contradiction. This means that $\left\|p_{i}^{n}(t)\right\|_{V_{i}^{*}} \rightarrow 0$ as $n \rightarrow \infty$ for a.e. $t \in(0, T)$ where $i=1$, 2 . Hence, the sequence $\left\{\mathbf{u}^{n}\right\}$ with $\mathbf{u}^{n}=\left(u_{1}^{n}, u_{2}^{n}\right)$ is an $\alpha$-approximating sequence for DSIP. So, it follows from the strong $\alpha$-well-posedness of DSIP that $\mathbf{u}^{\mathrm{n}}(t) \rightarrow \mathbf{u}(t)$ in $V$ for a.e. $t \in(0, T)$. Therefore, the system of time-dependent hemivariational inequalities STDHVI is strongly $\alpha$-well-posed. This completes the proof.

Utilizing the similar arguments to those in the proof of Theorem 4.6, on can easily prove the following equivalence between the strong $\alpha$-well-posedness in the generalized sense of STDHVI and the strong $\alpha$-well-posedness in the generalized sense of the system DSIP. 
Theorem 4.7. Let locally Lipschitz functional $\mathrm{J}(\mathrm{t}, \cdot \cdot \cdot)$ ) be regular on $\mathrm{X}$ for a.e. $\mathrm{t} \in(0, \mathrm{~T})$. Then, the system STDHVI of time-dependent hemivariational inequalities is strongly $\alpha$-well-posed in the generalized sense if and only if the derived system DSIP of inclusion problems is strongly $\alpha$-well-posed in the generalized sense.

Remark 4.8. Compared with [32, Theorems 4.5-4.6], our Theorems 4.6 and 4.7 use the system STDHVI of time-dependent hemivariational inequalities in place of the system SHVI of hemivariational inequalities, the derived system DSIP corresponding to STDHVI in place of the derived system DSIP corresponding to SHVI and the $\alpha$-well-posedness (resp., the $\alpha$-well-posedness in the generalized sense) in place of the well-posedness (resp., the well-posedness in the generalized sense), respectively. All in all, our Theorems 4.6 and 4.7 improve, extend and develop [32, Theorems 4.5-4.6] to a great extent.

\section{Concluding remarks}

The present paper generalizes the concept of $\alpha$-well-posedness to a system STDHVI of time-dependent hemivariational inequalities without Volterra integral terms in Banach spaces. We give several definitions of $\alpha$-well-posedness and, with assumptions (HAB), (HJ) on the operators involved in STDHVI, give some metric characterizations of $\alpha$-well-posedness for STDHVI considered. On the other hand, by establishing an equivalence result between the system STDHVI and a derived system DSIP of inclusion problems, we prove that the strong $\alpha$-well-posedness (resp., in the generalized sense) of STDHVI is equivalent to the strong $\alpha$-well-posedness (resp., in the generalized sense) of DSIP.

Several problems related to the $\alpha$-well-posedness of systems of time-dependent hemivariational inequalities remain to be considered in the future study. The first one is to exploit some conditions under which the strong (resp., weak) $\alpha$-well-posedness of systems of time-dependent hemivariational inequalities is equivalent to the existence and uniqueness of their solutions. The second one is to generalize the study of well-posedness (including $\alpha$-well-posedness) to systems of time-dependent hemivariational inequalities involving both nonsmooth functionals and proper, convex and lower semicontinuous functionals, which are referred to as systems of time-dependent variational-hemivariational inequalities. In addition, it is well-known that there are many other concepts of well-posedness in the literature on optimization problems and variational inequalities, such as well-posedness by perturbations $[2,5]$ and LevitinPolyak well-posedness $[3,7,18,19]$. Without question, extending these concepts of well-posedness to the study of systems of time-dependent hemivariational inequalities is very interesting and quite valuable in the future.

\section{Acknowledgment}

This research was partially supported by the Innovation Program of Shanghai Municipal Education Commission (15ZZ068), Ph.D. Program Foundation of Ministry of Education of China (20123127110002) and Program for Outstanding Academic Leaders in Shanghai City (15XD1503100). Yeong-Cheng Liou was supported in part by the grand form Kaohsiung Medical University Research Foundation (KMU-Q106005) and Taiwan-Russian joint grant MOST 106-2923-E-039-001-MY3. Jen-Chih Yao was partially supported by the Grant MOST 106-2923-E-039-001-MY3.

\section{References}

[1] S. Carl, V. K. Le, D. Motreanu, Nonsmooth variational problems and their inequalities, Comparison principles and applications, Springer Monographs in Mathematics, Springer, New York, (2007). 1, 2.6

[2] L.-C. Ceng, H. Gupta, C.-F. Wen, Well-posedness by perturbations of variational-hemivariational inequalities with perturbations, Filomat, 26 (2012), 881-895. 1, 5

[3] L.-C. Ceng, N. Hadjisavvas, S. Schaible, J. C. Yao, Well-posedness for mixed quasivariational-like inequalities, J. Optim. Theory Appl., 139 (2008), 109-125. 1, 5

[4] L.-C. Ceng, Y. C. Lin, Metric characterizations of $\alpha$-well-posedness for a system of mixed quasivariational-like inequalities in Banach spaces, J. Appl. Math., 2012 (2012), 22 pages. 3 
[5] L.-C. Ceng, C.-F. Wen, Well-posedness by perturbations of generalized mixed variational inequalities in Banach spaces, J. Appl. Math., 2012 (2012), 38 pages. 5

[6] L.-C. Ceng, N.-C. Wong, J.-C. Yao, Well-posedness for a class of strongly mixed variational-hemivariational inequalities with perturbations, J. Appl. Math., 2012 (2012), 21 pages. 1

[7] L. C. Ceng, J. C. Yao, Well-posedness of generalized mixed variational inequalities, inclusion problems and fixed-point problems, Nonlinear Anal., 69 (2008), 4585-4603. 5

[8] J.-W. Chen, Y. J. Cho, X.-Q. Ou, Levitin-Polyak well-posedness for set-valued optimization problems with constraints, Filomat, 28 (2014), 1345-1352. 1

[9] F. H. Clarke, Optimization and nonsmooth analysis, Second edition, Classics in Applied Mathematics, Society for Industrial and Applied Mathematics (SIAM), Philadelphia, PA, (1990). 2

[10] N. Costea, V. Rădulescu, Hartman-Stampacchia results for stably pseudomonotone operators and non-linear hemivariational inequalities, Appl. Anal., 89 (2010), 175-188. 1, 3

[11] Y.-P. Fang, R. Hu, N.-J. Huang, Well-posedness for equilibrium problems and for optimization problems with equilibrium constraints, Comput. Math. Appl., 55 (2008), 89-100. 1

[12] F. Giannessi, A. A. Khan, Regularization of non-coercive quasi variational inequalities, Control Cybernet., 29 (2000), 91-110. 1

[13] D. Goeleven, D. Mentagui, Well-posed hemivariational inequalities, Numer. Funct. Anal. Optim., 16 (1995), $909-921$. 1

[14] R. Hu, Y.-P. Huang, N.-J. Huang, M.-M. Wong, Well-posedness of systems of equilibrium problems, Taiwanese J. Math., 14 (2010), 2435-2446. 1

[15] X. X. Huang, X. Q. Yang, Generalized Levitin-Polyak well-posedness in constrained optimization, SIAM J. Optim., 17 (2006), 243-258. 1

[16] K. Kuratowski, Topology, Vol. II, New edition, revised and augmented, Translated from the French by A. Kirkor, Academic Press, New York-London; Państwowe Wydawnictwo Naukowe Polish Scientific Publishers, Warsaw, (1968). 2.10, 2

[17] B. Lemaire, Well-posedness, conditioning and regularization of minimization, inclusion and fixed-point problems, Proceedings of the 4th International Conference on Mathematical Methods in Operations Research and 6th Workshop on Well-posedness and Stability of Optimization Problems, Sozopol, (1997), Pliska Stud. Math. Bulgar., 12 (1998), 71-84. 1

[18] E. S. Levitin, B. T. Polyak, Convergence of minimizing sequences in problems on the relative extremum, (Russian) Dokl. Akad. Nauk SSSR, 168 (1966), 997-1000. 5

[19] X.-B. Li, F.-Q. Xia, Levitin-Polyak well-posedness of a generalized mixed variational inequality in Banach spaces, Nonlinear Anal., 75 (2012), 2139-2153. 5

[20] M. B. Lignola, Well-posedness and L-well-posedness for quasivariational inequalities, J. Optim. Theory Appl., 128 (2006), 119-138. 1

[21] L.-J. Lin, C.-S. Chuang, Well-posedness in the generalized sense for variational inclusion and disclusion problems and well-posedness for optimization problems with constraint, Nonlinear Anal., 70 (2009), 3609-3617. 1

[22] Z.-H. Liu, Browder-Tikhonov regularization of non-coercive evolution hemivariational inequalities, Inverse Problems, 21 (2005), 13-20. 1

[23] R. Lucchetti, F. Patrone, A characterization of Tyhonov well-posedness for minimum problems, with applications to variational inequalities, Numer. Funct. Anal. Optim., 3 (1981), 461-476. 1

[24] S. Migórski, A. Ochal, M. Sofonea, Nonlinear inclusions and hemivariational inequalities, Models and analysis of contact problems, Advances in Mechanics and Mathematics, Springer, New York, (2013). 1, 2, 2.12, 4

[25] D. Motreanu, P. D. Panagiotopoulos, Minimax theorems and qualitative properties of the solutions of hemivariational inequalities, Nonconvex Optimization and its Applications, Kluwer Academic Publishers, Dordrecht, (1999). 1

[26] Z. Naniewicz, P. D. Panagiotopoulos, Mathematical theory of hemivariational inequalities and applications, Monographs and Textbooks in Pure and Applied Mathematics, Marcel Dekker, Inc., New York, (1995). 1, 2

[27] P. D. Panagiotopoulos, Nonconvex energy functions, Hemivariational inequalities and substationarity principles, Acta Mech., 48 (1983), 111-130. 1

[28] P. D. Panagiotopoulos, Hemivariational inequalities, Applications in mechanics and engineering, Springer-Verlag, Berlin, (1993). 1

[29] P. D. Panagiotopoulos, M. Fundo, V. Rădulescu, Existence theorems of Hartman-Stampacchia type for hemivariational inequalities and applications, J. Global Optim., 15 (1999), 41-54. 1

[30] D. Repovš, C. Varga, A Nash type solution for hemivariational inequality systems, Nonlinear Anal., 74 (2011), 55855590. 1

[31] A. N. Tikhonov, On the stability of the functional optimization problem, USSR J. Comput. Math. Math. Phys., 6 (1966), 28-33. 1

[32] Y.-M. Wang, Y.-B. Xiao, X. Wang, Y. J. Cho, Equivalence of well-posedness between systems of hemivariational inequalities and inclusion problems, J. Nonlinear Sci. Appl., 9 (2016), 1178-1192. 1, 3, 3.6, 3.7, 3.13, 4.8

[33] Y.-B. Xiao, N.-J. Huang, Browder-Tikhonov regularization for a class of evolution second order hemivariational inequalities, J. Global Optim., 45 (2009), 371-388.1 
[34] Y.-B. Xiao, N.-J. Huang, Well-posedness for a class of variational-hemivariational inequalities with perturbations, J. Optim. Theory Appl., 151 (2011), 33-51. 1

[35] Y.-B. Xiao, N.-J. Huang, J. Lu, A system of time-dependent hemivariational inequalities with Volterra integral terms, J. Optim. Theory Appl., 165 (2015), 837-853. 1

[36] Y.-B. Xiao, N.-J. Huang, M.-M. Wong, Well-posedness of hemivariational inequalities and inclusion problems, Taiwanese J. Math., 15 (2011), 1261-1276. 1

[37] Y.-H. Yao, M. A. Noor, Y.-C. Liou, S. M. Kang, Iterative algorithms for general multivalued variational inequalities, Abstr. Appl. Anal., 2012 (2012), 10 pages. 1

[38] Y.-H. Yao, N. Shahzad, An algorithmic approach to the split variational inequality and fixed point problem, J. Nonlinear Convex Anal., 18 (2017), 977-991.

[39] H. Zegeye, N. Shahzad, Y.-H. Yao, Minimum-norm solution of variational inequality and fixed point problem in Banach spaces, Optimization, 64 (2015), 453-471. 1

[40] E. Zeidler, Nonlinear functional analysis and its applications, Translated from the German by the author and Leo F. Boron, Springer-Verlag, New York, (1990). 2

[41] Y.-L. Zhang, Y.-R. He, On stably quasimonotone hemivariational inequalities, Nonlinear Anal., 74 (2011), 3324-3332. 1

[42] T. Zolezzi, Extended well-posedness of optimization problems, J. Optim. Theory Appl., 91 (1996), 257-266. 1 